\title{
Ligand-Field-Modulated Molecular Junctions: On Covalently Bonded Ethynyl-Electrode Interfaces
}

\author{
Chih-Hsun Lin ${ }^{\dagger, \S}$ Geng-Min Lin, ${ }^{\dagger, \S}$ Er-Chien Horng, ${ }^{\dagger}$ Tzu-Ching Chou, ${ }^{\dagger}$ Yi-Chen Liu, ${ }^{\ddagger}$ Tsai-Hui \\ Wang, ${ }^{\ddagger}$ Wei-Ling Hua, ${ }^{\ddagger}$ Hsiu-Fu Hsu, ${ }^{*}$, and Chun-hsien Chen ${ }^{*}{ }^{\dagger}$ \\ 'Department of Chemistry and Center for Emerging Material and Advanced Device, National Taiwan University, Taipei, \\ Taiwan 10617. \\ tDepartment of Chemistry, Tamkang University, New Taipei City, Taiwan 25137. \\ ${ }^{\S}$ Contributed equally to this work.*e-mail: hhsu@mail.tku.edu.tw; chhchen@ntu.edu.tw
}

1. Research design. S3

1-1. Suitable headgroups to illustrate the ligand-field effect: $\mathrm{HCC}-\mathrm{X}-\mathrm{CCH}$ or $\mathrm{NC}-\mathrm{X}-\mathrm{CCH}$..... S3

Figure S1. Transmission spectra of $\mathrm{HCC}-\mathrm{X}-\mathrm{CCH}$ and $\mathrm{NC}-\mathrm{X}-\mathrm{CCH}(\mathrm{X}=\mathrm{OPE} 2$ or $\mathrm{C} 4)$........... S4

1-2. The strength of ligand-field effects on the degree of energy-level alignment. ...................... S5

Figure S2. The energy gap between HOMO and the average energy of $\mathrm{H}-\mathrm{C} \equiv \mathrm{C}-\mathrm{Au}$ and

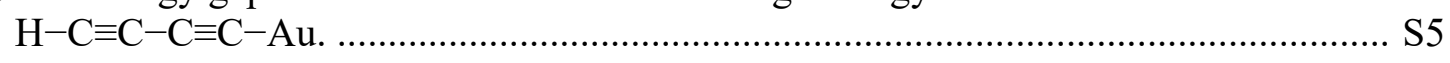

2. Synthesis and identification of model compounds. ......................................................................... S6

SchemeS1. Synthetic scheme of NC-OPE2-CCH, NC-OPE2-CC-AuPPh 3 , $\mathrm{NC}-\mathrm{OPE} 2-\mathrm{CC}-\mathrm{CCH}, \mathrm{NC}-\mathrm{OPE} 2-\mathrm{CC}-\mathrm{CC}-\mathrm{AuPPh}_{3}$, and $\mathrm{NC}-\mathrm{C} 4-\mathrm{CC}-\mathrm{AuPPh}_{3} . . . . . \mathrm{S} 6$

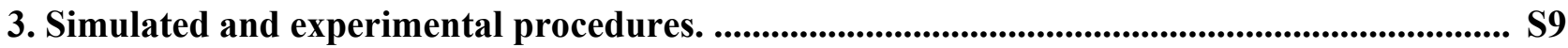

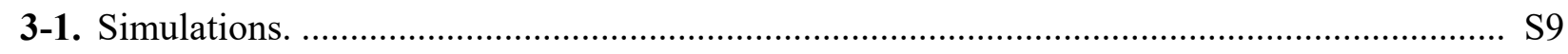

Figure S3. Illustration of the junction setup for the simulations of a molecule between

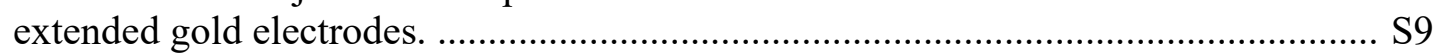

3-2. STM BJ-based single-molecule $i-V_{\text {bias }}$ measurements. .................................................... S10

Figure S4. Junction conductance as a function of the piezoelectric movement and the applied $V_{\text {bias }}$ for the measurements of STM BJ and $i-V_{\text {bias }}$ scans. ................................................... S1 1

3-3. Dependency of $\boldsymbol{i}-\boldsymbol{V}_{\text {bias }}$ curves on the $\boldsymbol{V}_{\text {bias }}$ scan rates. .................................................. S12

Figure S5. Overlaid $i-V_{\text {bias }}$ curves for $\mathrm{NC}-\mathrm{OPE} 2-\mathrm{CC}-\mathrm{CC}-\mathrm{AuPPh}_{3}$ as a function of scan rate. ... $\mathrm{S} 12$ 3-4. Probabilities of junction formation for molecules terminated by $-\mathrm{CCH}$ and $-\mathrm{CC}-\mathrm{AuPPh}_{3}$... S13

Figure S6. Conductance histograms of $\mathrm{NC}-\mathrm{OPE} 2-\mathrm{CC}-\mathrm{CC}$ junctions. .................................... S13

Figure S7. Molecular conductance histograms of $\mathrm{NC}-\mathrm{OPE} 2-\mathrm{CC}-\mathrm{CC}, \mathrm{NC}-\mathrm{OPE} 2-\mathrm{CC}$,

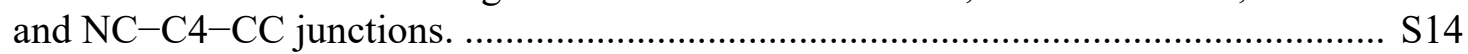

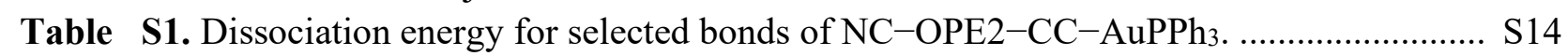

4. Peak assignment for transmission spectra. ........................................................................... S15

4-1. Peaks $1-4$ in the transmission spectra of $\mathrm{NC}-\mathrm{OPE} 2-\mathrm{CC}$ junctions. ..................................... S15

Figure S8. Isosurface plots of $\mathrm{NC}-\mathrm{OPE} 2-\mathrm{CC}$ junctions and $\mathrm{NC}-\mathrm{OPE} 2-\mathrm{CCH}$. ........................ S15

4-2. Orbitals facilitating the electron transport at $-\mathrm{CC}-\mathrm{Au}$ contacts. ........................................ S $\mathrm{S} 16$

Figure S9. Transmission spectra and isosurface plots of $\mathrm{NC}-\mathrm{C} 4-\mathrm{CC}$...................................... S16 
5. Simulated junction characteristics on pyramidal, stepped, and planar electrodes.

Figure S10. Three electrode geometries for the simulations of junction characteristics.

Figure S11. Zoomed transmission spectra at $V_{\text {bias }}=-0.7 \mathrm{~V}$ for NC-OPE2-CC-CC junctions. .. S17

Figure S12. PDOS plots for $\mathrm{NC}-\mathrm{OPE} 2-\mathrm{CC}$ and $\mathrm{NC}-\mathrm{C} 4-\mathrm{CC}$ junctions. ................................ S18

Figure S13. Effect of electrode geometry and $V_{\text {bias }}$ on the shift of $-\mathrm{CC}-\mathrm{Au} \pi_{\mathrm{x}}-d$ orbital. ......... S19

Figure S14. Effect of electrode geometry on simulated $i-V_{\text {bias }}$ curves and rectification ratios.... S20

6. Data processing for the experimental $i-V_{\text {bias }}$ curves.

S21

Figure S15. Treatment of the experimental $i-V_{\text {bias }}$ curves of $\mathrm{NC}-\mathrm{OPE} 2-\mathrm{CC}-\mathrm{CC}$ 


\section{Research design}

\section{1-1. Suitable headgroups to illustrate the ligand-field effect: $\mathrm{HCC}-\mathrm{X}-\mathrm{CCH}$ or $\mathrm{NC}-\mathrm{X}-\mathrm{CCH}$.}

To study how the ligand-field effect (LFE) improves the junction conductance, it would be straightforward to have a transmission peak which is associated with the LFE and can be readily identified. Regarding $\mathrm{CC}-\mathrm{OPE} 2-\mathrm{CC}$ junctions, the introductory section of the main text addresses that "for $\mathrm{Au}-\mathrm{CC}-\mathrm{OPE} 2-\mathrm{CC}-\mathrm{Au}$ junctions, the contribution of two $-\mathrm{CC}-\mathrm{Au}$ termini overlaps with the HOMO-1 peak at approximately $-0.7 \mathrm{eV}$ (Figure S1a), making the subsequent interpretation sophisticated". To illustrate this statement, Figure $\mathrm{S} 1 \mathrm{a}$ is prepared. The $-\mathrm{CC}-\mathrm{Au}$ interactions (indicated by $\dagger$ and $\ddagger$ with red arrows) are embedded in the HOMO and HOMO-1 peaks; the isosurface plots for $t$ and $\dagger$ reveal the strengths of $d_{\mathrm{xy}}$ (from the apical $\mathrm{Au}$ atom) relative to the molecule are more pronounced than those of HOMO and HOMO-1 peaks. In addition, the contribution to the orbital mixing from the $d$-orbitals of the second layer (i.e., the three gold atoms) for $\dagger$ and $\ddagger$ are more significant than for HOMO and HOMO-1 peaks. This overlaid problem is circumvented by studying NC-OPE2-CC junctions, in which only one of the termini is ethynyl-based and the $-\mathrm{CC}-\mathrm{Au}$ peak at $-0.7 \mathrm{eV}$ is not mixed with other peaks (the bottom panel of Figure S1a). Similarly, Figure S1b shows that the $-\mathrm{CC}-\mathrm{Au}$ degeneracy for $\mathrm{CC}-\mathrm{C} 4-\mathrm{CC}$ junctions is reduced to a single peak when one of the terminal groups becomes NC. Hence, in this LFE study, asymmetric terminal groups of $-\mathrm{CC}-\mathrm{Au}$ and $\mathrm{NC}-$ are employed for the model compounds. 


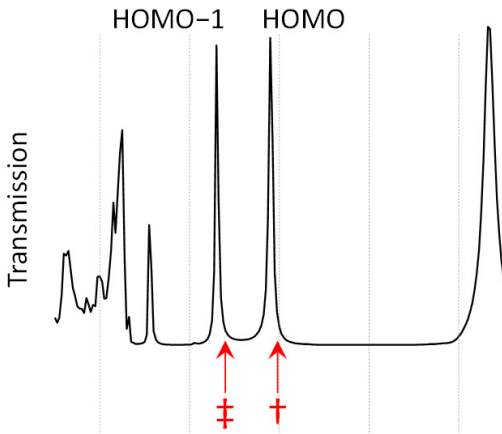

CC-OPE2-CC

HOMO

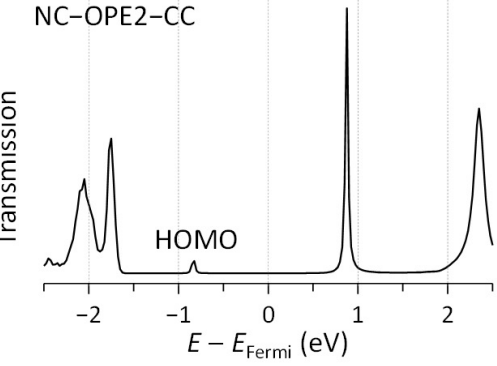

HOMO-1

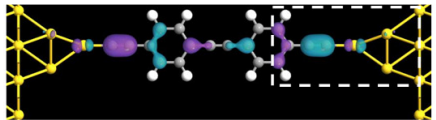

$\ddagger$
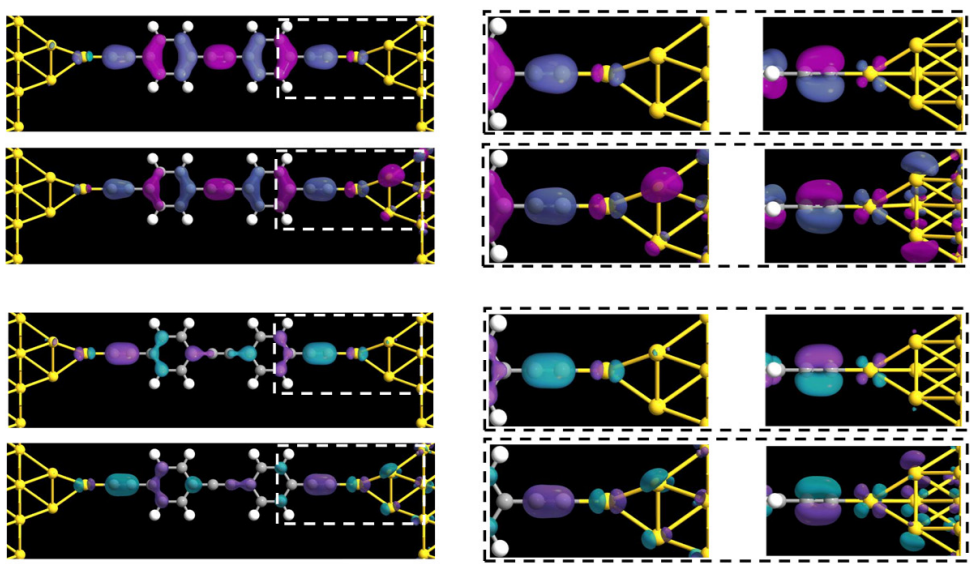

NC-OPE2-CC HOMO
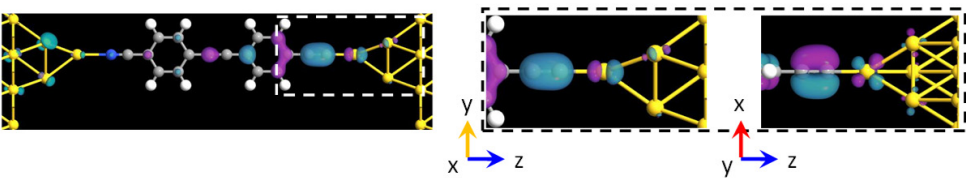

b $\quad \mathrm{CC}-\mathrm{C} 4-\mathrm{CC}$

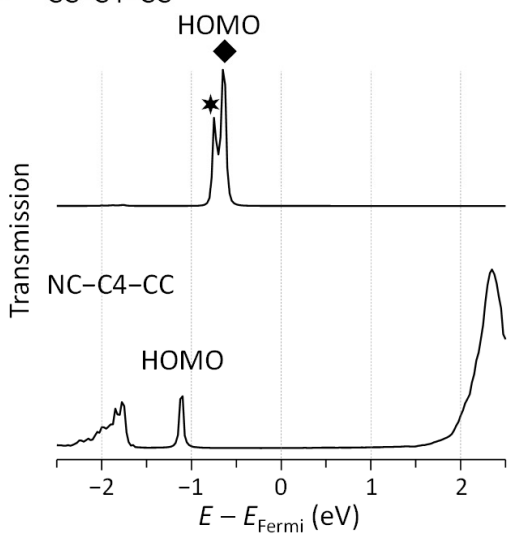

$\mathrm{CC}-\mathrm{C} 4-\mathrm{CC}$ HOMO *
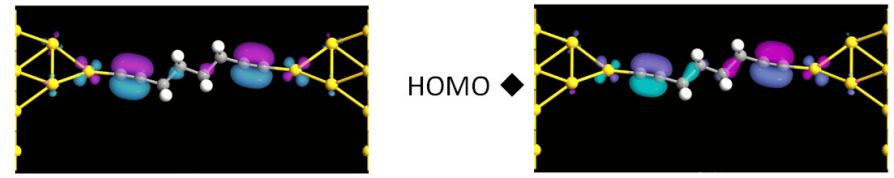

$\mathrm{NC}-\mathrm{C} 4-\mathrm{CC}$ HOMO
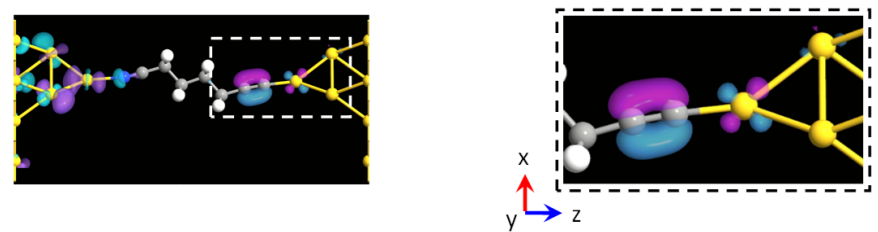

Figure S1. Transmission spectra of $\mathrm{HCC}-\mathrm{X}-\mathrm{CCH}$ and $\mathrm{NC}-\mathrm{X}-\mathrm{CCH}$ with $\mathrm{X}=(\mathrm{a}) \mathrm{OPE} 2$ or (b) $\mathrm{C} 4$. For $\mathrm{CC}-\mathrm{OPE} 2-\mathrm{CC}$ junctions, the contribution of $-\mathrm{CC}-\mathrm{Au}(\dagger$ and $\ddagger$ ) is embedded in the peaks of HOMO and $\mathrm{HOMO}-1$. For NC-OPE2-CC junctions, the characteristics $d_{\mathrm{xy}}$ of gold for $-\mathrm{CC}-\mathrm{Au}$ is found at $\sim 0.7 \mathrm{eV}$ and is not complicated by other orbitals. For $\mathrm{C} 4$ backbones, $\mathrm{CC}-\mathrm{C} 4-\mathrm{CC}$ junctions exhibit degenerate peaks $*$ and $\bullet$. $\mathrm{NC}-\mathrm{C} 4-\mathrm{CC}$ junctions show only one HOMO peak of $-\mathrm{CC}-\mathrm{Au}$ interactions. The contribution of the $d_{\mathrm{xy}}$ of gold for $-\mathrm{CC}-\mathrm{Au}$ moiety is referred to Figure 1 (main text) and Figure S2. For OPE2 junctions, there are two sets of viewing angles to provide better observations for (middle column) OPE moiety and (right column) the $d_{\mathrm{xz}}$ orbital of the apical gold atom. Please be noted that the intensities of the molecular moiety relative to that of gold atoms (particularly the second layer) are significantly different for the OPE2 junctions. The spectra were simulated under zero bias. 


\section{1-2. The strength of ligand-field effects on the degree of energy-level alignment.}

Figure 1 of the main text shows portions of gas-phase orbitals interaction diagrams to suggest that stronger headgroup-electrode interactions can lead to a better energy-level alignment. The value of $\mid E_{\text {Fermi }}$ - Eномо is $1.9 \mathrm{eV}$ for $\mathrm{H}-\mathrm{CC}-\mathrm{Au}$ and $1.5 \mathrm{eV}$ for $\mathrm{H}-\mathrm{CC}-\mathrm{CC}-\mathrm{Au}$. Figure $\mathrm{S} 2$ presents more detailed diagrams. Note that $\pi_{\mathrm{x}}$ and $\pi_{\mathrm{y}}$ of the ethynyl group are degenerate, and so are $d_{\mathrm{xz}}$ and $d_{\mathrm{yz}}$ orbitals of the $\mathrm{Au}$ atom. Hence, with a 90-degree rotation, Panels a and b are identical to Panels $\mathrm{c}$ and $\mathrm{d}$, respectively.

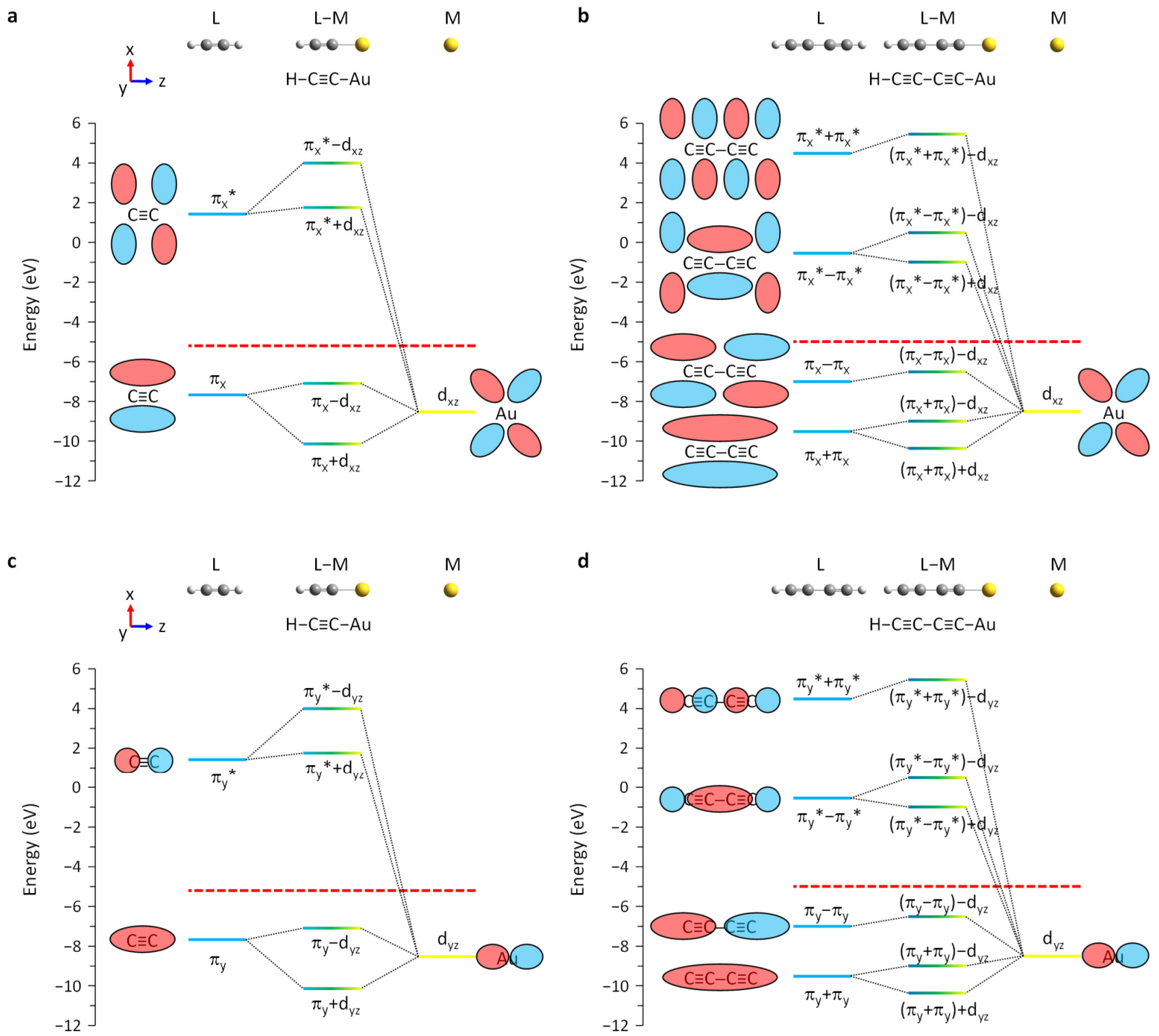

Figure S2. The energy gap between HOMO and the average energy (the red dash lines, viz., $\left.E_{\mathrm{Fermi}}\right)^{1-3}$ is $0.4 \mathrm{eV}$ smaller for $(\mathrm{a}, \mathrm{c}) \mathrm{H}-\mathrm{C} \equiv \mathrm{C}-\mathrm{Au}$ than for $(\mathrm{b}, \mathrm{d}) \mathrm{H}-\mathrm{C} \equiv \mathrm{C}-\mathrm{C} \equiv \mathrm{C}-\mathrm{Au}$ with the orbital mixing of (upper) $\pi_{\mathrm{x}}-d_{\mathrm{xz}}$ and (lower) $\pi_{\mathrm{y}}-d_{\mathrm{yz}}$. For a terse presentation, the coefficients of the $\pi_{\mathrm{y}}$ and $d$ orbitals are omitted. For the orbital mixing between $\pi^{*}$ and $d$ orbitals (viz., those above the average energy), the coefficient of $d_{\mathrm{xz}}$ is presumably very small considering the relative energy difference between these orbitals. Intriguingly, the component of gold is found from the isosurface plots. Average energy: $\mathrm{H}-\mathrm{C} \equiv \mathrm{C}-\mathrm{Au}$, $-5.2 \mathrm{eV} ; \mathrm{H}-\mathrm{C} \equiv \mathrm{C}-\mathrm{C} \equiv \mathrm{C}-\mathrm{Au},-5.0 \mathrm{eV} ; E_{\mathrm{HOMO}}:\left(\pi_{\mathrm{x}}-d_{\mathrm{xz}}\right)$ and $\left(\pi_{\mathrm{y}}-d_{\mathrm{yz}}\right),-7.1 \mathrm{eV} ;\left(\left(\pi_{\mathrm{x}}-\pi_{\mathrm{x}}\right)-d_{\mathrm{xz}}\right)$ and $\left(\left(\pi_{\mathrm{y}}-\pi_{\mathrm{y}}\right)-d_{\mathrm{yz}}\right),-6.5 \mathrm{eV}$. The simulations were performed by Gaussian09 using B3LYP/LAN2DZ/6-31G*. 


\section{Synthesis and identification of model compounds}

Compounds $\mathrm{NC}-\mathrm{OPE} 2-\mathrm{CCH}$ and $\mathrm{NC}-\mathrm{OPE} 2-\mathrm{CC}-\mathrm{AuPPh}_{3}$ were reported in literature and were prepared by the literature procedures. ${ }^{4}$ Compound $\mathrm{NC}-\mathrm{C} 4-\mathrm{CCH}$ was obtained from TCI and used as received. Compound $\mathrm{NC}-\mathrm{C} 4-\mathrm{CC}-\mathrm{AuPPh}_{3}$ was prepared herein by adopting the preparation ${ }^{4}$ of $\mathrm{NC}-\mathrm{OPE} 2-\mathrm{CC}-\mathrm{AuPPh}_{3}$.

\section{Synthesis of model compounds.}
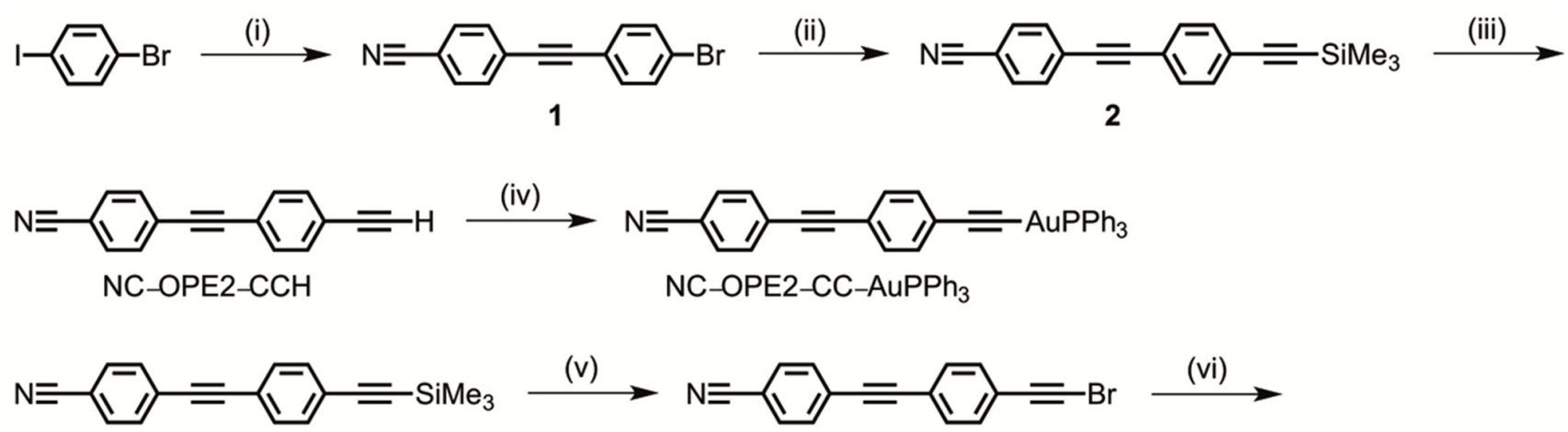

2

3
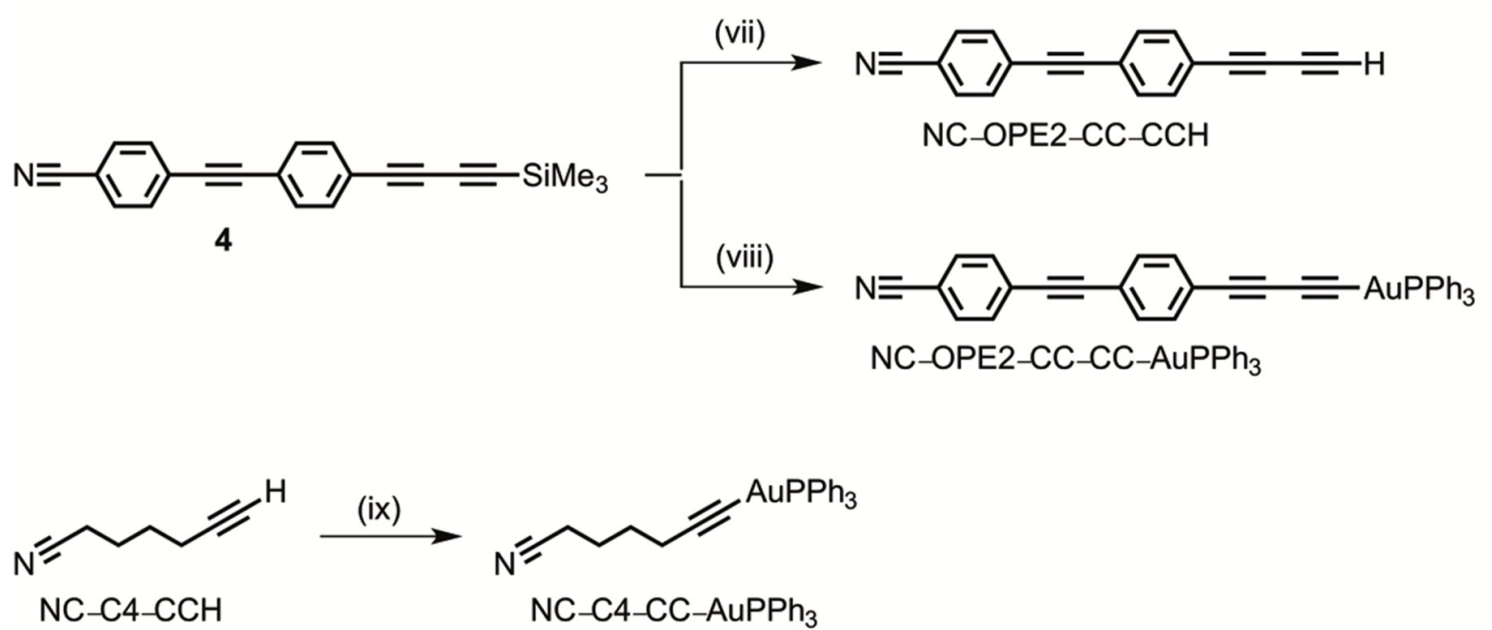

Scheme S1. Synthetic scheme of $\mathrm{NC}-\mathrm{OPE} 2-\mathrm{CCH}, \mathrm{NC}-\mathrm{OPE} 2-\mathrm{CC}-\mathrm{AuPPh}_{3}, \mathrm{NC}-\mathrm{OPE} 2-\mathrm{CC}-\mathrm{CCH}$, $\mathrm{NC}-\mathrm{OPE} 2-\mathrm{CC}-\mathrm{CC}-\mathrm{AuPPh}_{3}$, and $\mathrm{NC}-\mathrm{C} 4-\mathrm{CC}-\mathrm{AuPPh}_{3}$. Conditions: (i) 4-ethynylbenzonitrile, $\mathrm{Pd}\left(\mathrm{PPh}_{3}\right)_{2} \mathrm{Cl}_{2}, \mathrm{PPh}_{3}, \mathrm{CuI}, \mathrm{Et}_{3} \mathrm{~N}$, room temperature; (ii) TMSA (trimethylsilylacetylene), $\mathrm{Pd}\left(\mathrm{PPh}_{3}\right)_{2} \mathrm{Cl}_{2}$, $\mathrm{PPh}_{3}, \mathrm{CuI}, \mathrm{Et}_{3} \mathrm{~N} / \mathrm{THF}$ (2/1), reflux; (iii) $\mathrm{K}_{2} \mathrm{CO}_{3}, \mathrm{MeOH} / \mathrm{THF}$ (1/1), room temperature; (iv) $\mathrm{NaOH}, \mathrm{MeOH}$, $\mathrm{AuClPPh}_{3}$, room temperature; (v) NBS, $\mathrm{KF}, \mathrm{AgNO}_{3}, \mathrm{MeCN}$, trace $\mathrm{H}_{2} \mathrm{O}$, room temperature; (vi) TMSA, $\mathrm{Pd}\left(\mathrm{PPh}_{3}\right)_{2} \mathrm{Cl}_{2}, \mathrm{PPh}_{3}, \mathrm{CuI}, \mathrm{Et}_{3} \mathrm{~N}$, reflux; (vii) $\mathrm{K}_{2} \mathrm{CO}_{3}, \mathrm{MeOH} / \mathrm{THF}$ (1/10), room temperature; (viii) $\mathrm{NaOH}$, $\mathrm{MeOH}, \mathrm{AuClPPh}_{3}$, room temperature; (ix) $\mathrm{NaOH}, \mathrm{MeOH}, \mathrm{AuClPPh}_{3}$, room temperature.

NC-OPE2-CCH: A mixture of Compound $2(30 \mathrm{mg}, 0.10 \mathrm{mmol})$ and $\mathrm{K}_{2} \mathrm{CO}_{3}(138 \mathrm{mg}, 1.0 \mathrm{mmol})$ in THF $(1.5 \mathrm{~mL})$ and $\mathrm{MeOH}(1.5 \mathrm{~mL})$ were stirred at room temperature for $4 \mathrm{~h}$. The reaction mixture was filtered and washed with dichloromethane, and then purified by column chromatography with dichloromethane and $\mathrm{MeOH}(1 / 3)$ to afford $\mathrm{NC}-\mathrm{OPE} 2-\mathrm{CCH}$ as a pale yellow solid (62\%). 


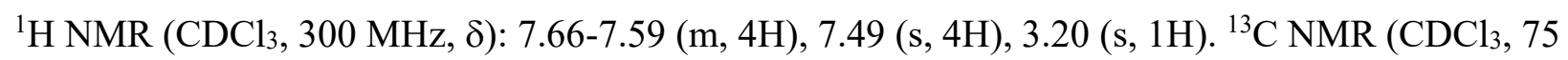
MHz, $\delta): 132.2,132.1,131.7,127.9,122.9,122.6,118.4,111.8,93.1,89.5,83.0,79.4$. HRMS-EI $(\mathrm{m} / \mathrm{z})$ : $[\mathrm{M}]^{+}$calcd for $\mathrm{C}_{17} \mathrm{H}_{9} \mathrm{~N}, 227.0735$; found, 227.0737 .

NC-OPE2-CC-AuPPh ${ }_{3}$ : A mixture of the corresponding diyne compound (100 mg, $0.44 \mathrm{mmol}$ ) and $\mathrm{NaOH}(88 \mathrm{mg}, 2.2 \mathrm{mmol})$ in $\mathrm{MeOH}(10 \mathrm{~mL})$ were stirred at room temperature for $30 \mathrm{~min}$, and then $\mathrm{AuClPPh}_{3}(218 \mathrm{mg}, 0.44 \mathrm{mmol})$ was added. The reaction mixture was stirred for further $5 \mathrm{~h}$ to form a white precipitate. The precipitate was filtered and washed with $\mathrm{MeOH}$, dichloromethane, and hexane and then dried to afford $\mathrm{NC}-\mathrm{OPE} 2-\mathrm{CC}-\mathrm{AuPPh}_{3}$ as a pale gray solid (57\%).

${ }^{1} \mathrm{H}$ NMR $\left(\mathrm{CD}_{2} \mathrm{Cl}_{2}, 600 \mathrm{MHz}, \delta\right): 7.66-7.40$ (m, 19H). ${ }^{13} \mathrm{C} \mathrm{NMR}\left(\mathrm{CD}_{2} \mathrm{Cl}_{2}, 150 \mathrm{MHz}, \delta\right): 134.7,134.6$ $\left(\mathrm{d}, J_{\mathrm{CP}}=13.5 \mathrm{~Hz}\right), 132.5,132.4,132.0,131.9,130.3,130.0,129.6,129.5\left(\mathrm{~d}, J_{\mathrm{CP}}=10.5 \mathrm{~Hz}\right), 128.5,126.8$, 120.5, 118.9, 111.8, 94.0, 89.2. HRMS-FAB $(m / z):[\mathrm{M}+\mathrm{H}]^{+}$calcd for $\mathrm{C}_{35} \mathrm{H}_{24} \mathrm{AuNP}, 686.1312$; found, 686.1310 .

NC-OPE2-CC-CCH: A mixture of Compound 4 (60 mg, $0.19 \mathrm{mmol})$ and $\mathrm{K}_{2} \mathrm{CO}_{3}(129 \mathrm{mg}, 0.93$ $\mathrm{mmol})$ in THF $(1.5 \mathrm{~mL})$ and $\mathrm{MeOH}(1.5 \mathrm{~mL})$ were stirred at room temperature for $1 \mathrm{~h}$. The reaction mixture was filtered and washed with dichloromethane, and then purified by recrystallization with dichloromethane and $\mathrm{MeOH}$ to afford $\mathrm{NC}-\mathrm{OPE} 2-\mathrm{CC}-\mathrm{CCH}$ as a brown solid (10\%).

${ }^{1} \mathrm{H} \mathrm{NMR}\left(\mathrm{CDCl}_{3}, 300 \mathrm{MHz}, \delta\right): 7.65(\mathrm{~d}, J=8.0 \mathrm{~Hz}, 2 \mathrm{H}), 7.60$ (d, $\left.J=8.5 \mathrm{~Hz}, 2 \mathrm{H}\right), 7.53-7.47$ (m, 4H), 2.56 (s, 1H). ${ }^{13} \mathrm{C} \mathrm{NMR}\left(\mathrm{CDCl}_{3}, 75 \mathrm{MHz}, \delta\right): 133.0,132.3,132.0,128.0,123.6,121.9,118.6,112.1,93.1$, 90.5, 75.9, 74.8, 72.7, 68.1. HRMS-EI ( $\mathrm{m} / \mathrm{z}):[\mathrm{M}]^{+}$calcd for $\mathrm{C}_{19} \mathrm{H}_{9} \mathrm{~N}, 251.0735$; found, 251.0733.

NC-OPE2-CC-CC-AuPPh $:$ A mixture of the corresponding diyne compound (50 mg, 0.16 $\mathrm{mmol})$ and $\mathrm{NaOH}(62 \mathrm{mg}, 1.5 \mathrm{mmol})$ in $\mathrm{MeOH}(1 \mathrm{~mL})$ were stirred at room temperature for $30 \mathrm{~min}$, and then $\mathrm{AuClPPh}_{3}(77 \mathrm{mg}, 0.16 \mathrm{mmol}$ ) was added. The reaction mixture was stirred for further $1.5 \mathrm{~h}$ to form a yellow precipitate. The precipitate was filtered and washed with dichloromethane, and then purified by recrystallization with dichloromethane and $\mathrm{MeOH}$ to afford $\mathrm{NC}-\mathrm{OPE} 2-\mathrm{CC}-\mathrm{CC}-\mathrm{AuPPh}_{3}$ as a yellow solid $(33 \%)$.

${ }^{1} \mathrm{H}$ NMR $\left(\mathrm{CD}_{2} \mathrm{Cl}_{2}, 600 \mathrm{MHz}, \delta\right): 7.66-7.62(\mathrm{~m}, 4 \mathrm{H})$, 7.57-7.45 (m, 19H). ${ }^{13} \mathrm{C} \mathrm{NMR}\left(\mathrm{CD}_{2} \mathrm{Cl}_{2}, 150\right.$ $\mathrm{MHz}, \delta): 134.7,134.6\left(\mathrm{~d}, J_{\mathrm{CP}}=13.6 \mathrm{~Hz}\right), 133.0,132.5,132.4,132.1,132.0,130.0,129.7,129.6\left(\mathrm{~d}, J_{\mathrm{CP}}=\right.$ $10.6 \mathrm{~Hz}), 128.2,124.2,122.3,118.8,112.1,93.5,90.1,86.0,78.7,69.9 .{ }^{31} \mathrm{P} \mathrm{NMR}\left(\mathrm{CD}_{2} \mathrm{Cl}_{2}, 242 \mathrm{MHz}, \delta\right)$ : 43.0. HRMS-FAB $(\mathrm{m} / z)$ : $[\mathrm{M}+\mathrm{H}]^{+}$calcd for $\mathrm{C}_{37} \mathrm{H}_{24} \mathrm{AuNP}$, 710.1312; found, 710.1324. Anal. Calcd for $\mathrm{C}_{37} \mathrm{H}_{23} \mathrm{AuNP}$ : C, 62.63; H, 3.27; Au, 27.76; N, 1.97; P, 4.37. Found: C, 62.18; H, 3.15; N, 2.22.

NC-C4-CC-AuPPh: A mixture of the corresponding diyne compound (50 $\mathrm{mg}, 0.47 \mathrm{mmol}$ ) and $\mathrm{NaOH}(47 \mathrm{mg}, 1.18 \mathrm{mmol})$ in $\mathrm{MeOH}(3 \mathrm{~mL})$ were stirred at room temperature for $30 \mathrm{~min}$, and then $\mathrm{AuClPPh}_{3}(231 \mathrm{mg}, 0.47 \mathrm{mmol})$ was added. The reaction mixture was stirred for further $3 \mathrm{~h}$ to form a 
white precipitate. The precipitate was filtered and washed with $\mathrm{MeOH}$ and hexane, and then purified by recrystallization with dichloromethane and $\mathrm{MeOH}$ to afford $\mathrm{NC}-\mathrm{C} 4-\mathrm{CC}-\mathrm{AuPPh}_{3}$ as a white solid (8\%).

${ }^{1} \mathrm{H}$ NMR $\left(\mathrm{CD}_{2} \mathrm{Cl}_{2}, 600 \mathrm{MHz}, \delta\right): 7.55-7.45(\mathrm{~m}, 15 \mathrm{H}), 2.38$ (t, $\left.J=7.2 \mathrm{~Hz}, 2 \mathrm{H}\right), 2.34(\mathrm{t}, J=6.9 \mathrm{~Hz}$, 2H), 1.85-1.80 (m, 2H), 1.65-1.60 (m, 2H). $\left.{ }^{13} \mathrm{C} \mathrm{NMR} \mathrm{(} \mathrm{CD}_{2} \mathrm{Cl}_{2}, 150 \mathrm{MHz}, \delta\right): 134.7,134.6$ (d, $J_{\mathrm{CP}}=13.5$ $\mathrm{Hz}), 131.9,130.6,130.2\left(\mathrm{~d}, J_{\mathrm{CP}}=54.3 \mathrm{~Hz}\right), 129.5,129.4\left(\mathrm{~d}, J_{\mathrm{CP}}=12.1 \mathrm{~Hz}\right), 120.2,28.9,25.0,19.3,17.1$. ${ }^{31} \mathrm{P}$ NMR $\left(\mathrm{CD}_{2} \mathrm{Cl}_{2}, 242 \mathrm{MHz}, \delta\right): 42.3$. HRMS-FAB $(m / z)$ : [M+H $]^{+}$calcd for $\mathrm{C}_{25} \mathrm{H}_{24} \mathrm{AuNP}, 566.1312$; found, 566.1303 . 


\section{Simulated and experimental procedures.}

\section{3-1. Simulations.}

For the calculations of energy-level diagrams for $\mathrm{H}-\mathrm{C} \equiv \mathrm{C}-\mathrm{Au}$ and $\mathrm{H}-\mathrm{C} \equiv \mathrm{C}-\mathrm{C} \equiv \mathrm{C}-\mathrm{Au}$, we adopted B3LYP/LAN2DZ/6-31G* implemented in the Gaussian 09 package. ${ }^{5}$ Simulations of electric transport were performed by utilizing the DFT-NEGF approach (Synopsys, QuantumATK 2017.2). ${ }^{6,7}$ The GGA/PBE functional (generalized gradient approximation/Perdew-Burke-Ernzerhof parameterization) ${ }^{8}$ was used as the exchange-correlation functional. A double- $\zeta$ polarization (DZP) basis set was employed for all elements. Figure S3 displays a schematic illustration of the molecular junction. The electrode extension region has 3 layers of $4 \times 4 \mathrm{Au}(111)$ on each electrode. In addition to the molecule, the central scattering region on each side contains 3 layers of $4 \times 4 \mathrm{Au}(111)$ (16 Au atoms per layer) and an electrode configuration to bridge the molecule. There were three types of surface electrode configurations, namely, the 20-atom gold pyramid (3 layers), 19-atom gold step (2 layers), and 16-atom planar (one layer) Au(111) (Figure S9). The initial values for $\mathrm{C}-\mathrm{Au}$ and $\mathrm{N}-\mathrm{Au}$ spacing were $2.00 \AA$ and $2.22 \AA$, respectively. ${ }^{4,9,10}$ To reduce the computational cost, the optimization with all forces $<0.05 \mathrm{eV}^{-1}$ was performed on the molecule and the contacted part of the electrodes. Specifically, the numbers of optimized gold atoms on each side were 4, 3, and 1 for the pyramidal, step, and planar configurations, respectively. The subsequent transmission spectra and PDOS were computed with $\mathrm{k}$-point meshes of $3 \times 3 \times 100$ and $3 \times 3 \times 1$, respectively.

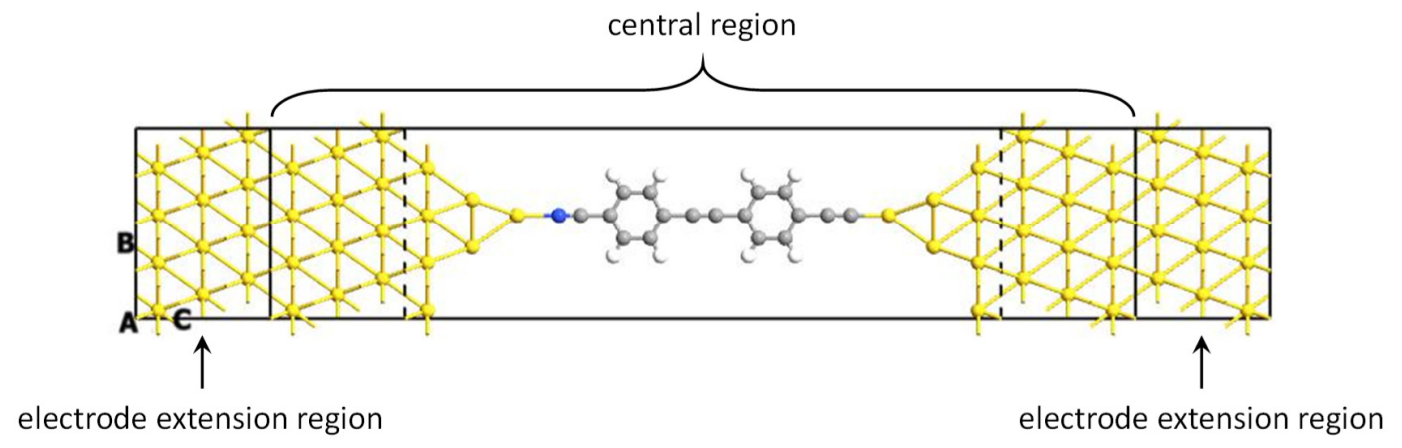

Figure S3. Illustration of the junction setup for the simulations of a molecule between extended gold electrodes. The central scattering region includes the molecule, the contact, and, on each electrode, 3 surface layers of $4 \times 4 \mathrm{Au}(111)$ slabs (between the vertical dashed and solid lines). Regarding the electrode configurations at the molecule-electrode contact, the pyramidal, stepped, and planar structures are constructed by 20 (3 layers), 19 (2 layers), and 16 (one layer) gold atoms, respectively. 


\section{3-2. STM BJ-based single-molecule $i-V_{\text {bias }}$ measurements.}

The STM tips were mechanically cut 0.25 - $\mathrm{mm}$ gold wires (99.95\%, Leesan, Tainan, Taiwan). The substrate was 100-nm thick gold on glass slides, prepared by thermal evaporation at a nominal pressure of $2 \times 10^{-6}$ Torr with ca. 5-nm chromium (99.99\%, Super Conductor Materials, Inc., Suffern, NY, USA) as the adhesion layer.

Experimental measurements were carried out with a NanoScope IIIa controller equipped with a signal access module (Veeco). A data acquisition system with a 24-bit PCI-4461 card driven by customized LabVIEW programs (National Instruments) was used for the control over the applied piezo voltages for the tip displacement as well as the $V_{\text {bias }}$ and current signal acquisition at a rate of 10,000 points/sec. ${ }^{11,12}$

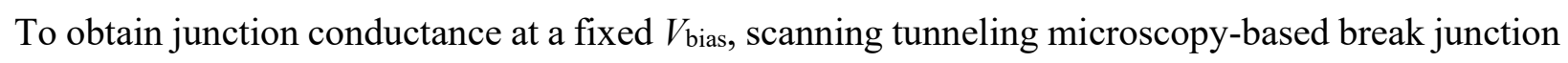
(STM BJ) was performed. The experiments were carried out with the STM tip and substrate immersed in mesitylene (ACROS) containing the target molecule under a typical $V_{\text {bias }}$ of $50 \mathrm{mV}$. The STM tip was repeatedly crashed into (Segment I of Figure S4b) and subsequently retracted from (Segment II of Figure $\mathrm{S} 4 \mathrm{~b}$ ) the substrate at a traveling distance of $10 \mathrm{~nm}$ and a traveling rate of $50 \mathrm{~nm} / \mathrm{sec}$ with a $0.1-\mathrm{sec}$ pause (Segment III of Figure S4b) between each cycle. Molecular junctions formed during tip retraction were detected as conductance steps below gold atom point contact, i.e. around $1.0 \mathrm{G}_{0}$. Conductance histograms were constructed from more than 5,000 retract traces without data selection to obtain statistical conductance value of the target molecule.

To acquire $i-V_{\text {bias }}$ curves, ${ }^{13-21}$ the measurement procedures were modified from the aforementioned algorithm with a feedback loop. At the start of each measurement cycle, the $V_{\text {bias }}$ was set at $50 \mathrm{mV}$. The STM tip was first set to crash into the substrate at a traveling rate of $50 \mathrm{~nm} / \mathrm{sec}$ (Segment I of Figure S4d). When the measured conductance corresponding to more than $10 \mu \mathrm{A}\left(\sim 2.8 \mathrm{G}_{0}\right)$ was detected, the tip was withdrawn from the substrate at a slower traveling rate of $\leq 3 \mathrm{~nm} / \mathrm{sec}$ (Section II of Figure S4d) until the measured conductance became smaller than $0.9 \mathrm{G}_{0}$. The tip was then held in position for $0.9 \mathrm{sec}$ (Segment III of Figure S4d), during which the $V_{\text {bias }}$ was sweep between $\pm 1.5 \mathrm{~V}$ for 2.5 cycles at a constant rate of $20 \mathrm{~V} / \mathrm{sec}$. Afterwards, the tip was further withdrawn until noise level (below $10^{-5} \mathrm{G}_{0}$ ) was reached (Segment IV of Figure S4d), during which the molecular junction broke down. There was a 0.1-sec interval between cycles. For the data treatment, only those exhibiting junction conduction without falling into the noise level during the 2.5 cycles were considered to be valid and were pooled to plot the $i-V_{\text {bias }}$ results of the target molecule. 

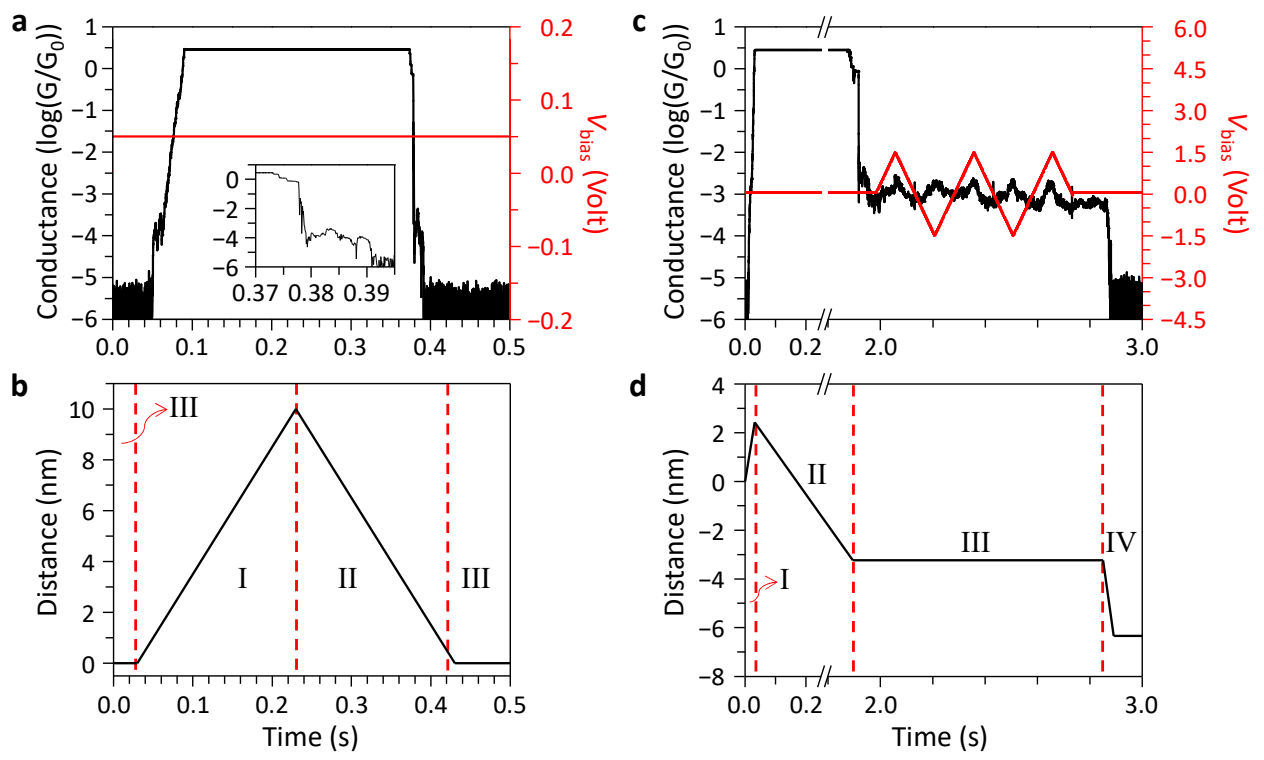

Figure S4. Junction conductance as a function of the piezoelectric movement and the applied $V_{\text {bias }}$ for the measurements of $(\mathrm{a}, \mathrm{b})$ STM BJ and $(\mathrm{c}, \mathrm{d}) i-V_{\text {bias }}$ scans. Panels a and $\mathrm{b}$ were acquired synchronously and thus they share the same time axis. So were Panels c and d. (a) A conductance trace (in black) was measured under a fixed tip-substrate $V_{\text {bias }}$ of $50 \mathrm{mV}$ (red line) while (c) the routine of the z-piezo was motionless (III), driving the tip approaching toward (I) and retracting from (II) the substrate. The inset of Panel a is a magnified section showing steps corresponding to gold-atom chains and molecular junctions. (c) A conductance trace (black trace) was measured under bias sweeping (red line, for 2.5 cycles) with (d) the motion of the z-piezo which contained four segments, approaching (I), retracting (II), motionless for the bias sweeping (III), and pre-programmed retracting to restart the next cycle (IV). The compound for this demonstration was $\mathrm{H}_{2} \mathrm{~N}\left(\mathrm{C}_{6} \mathrm{H}_{4}\right)_{3} \mathrm{NH}_{2}$. 


\section{3-3. Dependency of $i-V_{\text {bias }}$ curves on the $V_{\text {bias }}$ scan rates}

To obtain $i-V_{\text {bias }}$ curves at the junction lifetime of $\leq 1 \mathrm{sec}$, a scan rate of $20 \mathrm{~V} / \mathrm{s}$ was employed. Such a fast ramping speed raises questions about whether the $i-V_{\text {bias }}$ characteristics is dependent on the scan rate. To clarify this issue, $i-V_{\text {bias }}$ experiments at $20 \mathrm{~V} / \mathrm{s}, 100 \mathrm{~V} / \mathrm{s}$ and $500 \mathrm{~V} / \mathrm{s}$ were carried out by the method described in the previous section (3-2 and Panels c and d of Figure S4). The results (Figure S5) show that the $i-V_{\text {bias }}$ curves are indistinguishable under the three scan rates although the most probable traces (the black lines) appear slightly more conductive for faster scans.
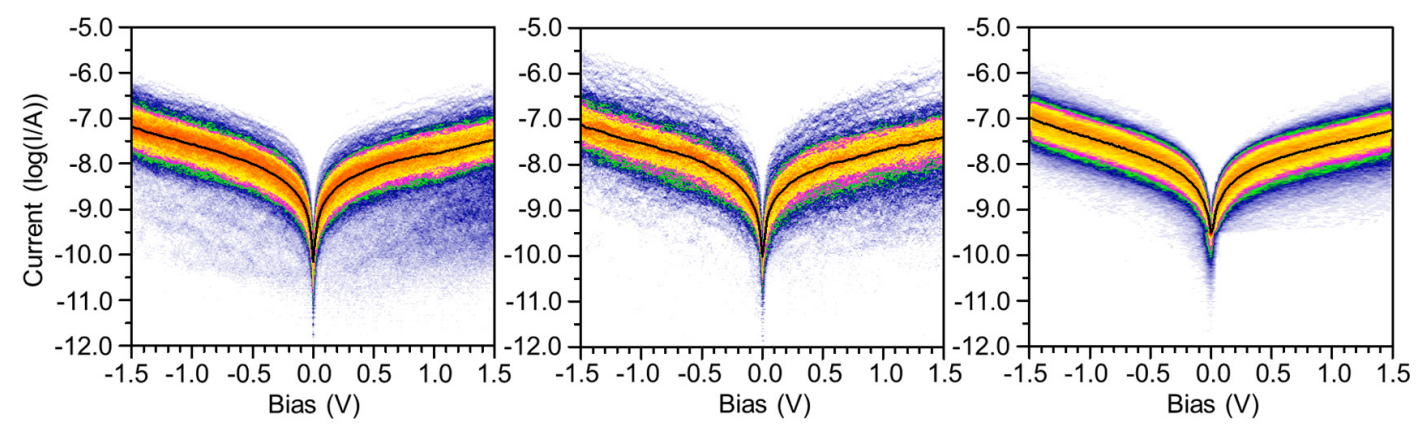

Figure S5. Overlaid $i-V_{\text {bias }}$ curves for $\mathrm{NC}-\mathrm{OPE} 2-\mathrm{CC}-\mathrm{CC}-\mathrm{AuPPh}_{3}$ as a function of scan rate. Scan rate of $V_{\text {bias: }}$ left, $20 \mathrm{~V} / \mathrm{s}$; middle, $100 \mathrm{~V} / \mathrm{s}$; right, $500 \mathrm{~V} / \mathrm{s}$. Black lines are the most probable $i-V_{\text {bias }}$ curves by Gaussian fitting for 1D histograms prepared at an interval of $20 \mathrm{mV}$. 


\section{3-4. Probabilities of junction formation for molecules terminated by $-\mathrm{CCH}$ and $-\mathrm{CC}-\mathrm{AuPPh}$.}

Panels $\mathrm{a}$ and $\mathrm{b}$ of Figure $\mathrm{S} 6$ are the $2 \mathrm{D}$ conductance histograms for $\mathrm{NC}-\mathrm{OPE} 2-\mathrm{CC}-\mathrm{CCH}$ and $\mathrm{NC}-\mathrm{OPE} 2-\mathrm{CC}-\mathrm{CC}-\mathrm{AuPPh}_{3}$, respectively. Their peak values are very close, suggesting that both compounds result in the same $\mathrm{NC}-\mathrm{OPE} 2-\mathrm{CC}-\mathrm{CC}$ junctions. The formation probability for $-\mathrm{CC}-\mathrm{AuPPh} 3$ is $40.5 \%$ (2047 out of 5050 traces), significantly higher than $15.8 \%$ (806 out of 5105 traces) of $-\mathrm{CCH}$. Therefore, $\mathrm{AuPPh}_{3}$-containing model compounds are employed for this study. Figure $\mathrm{S} 7$ shows the molecular conductance histograms of $\mathrm{AuPPh}_{3}$-containing model compounds.
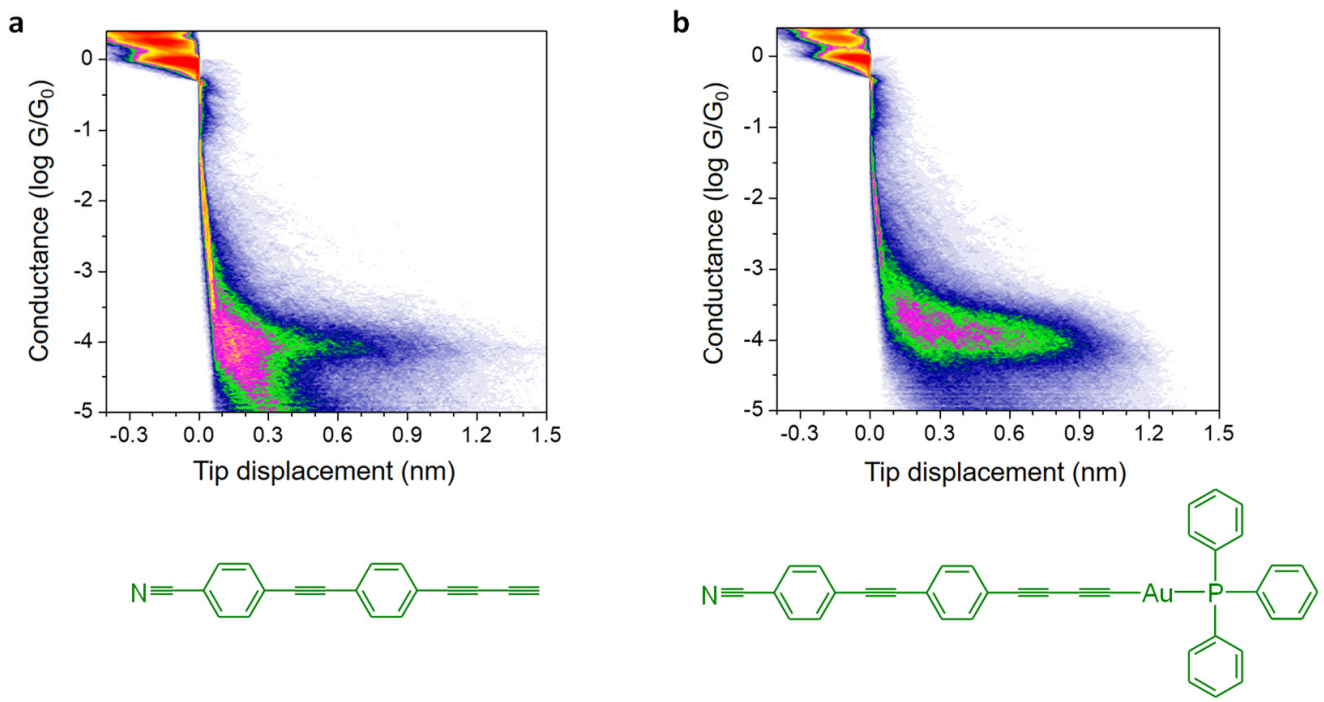

Figure S6. Conductance histograms of $\mathrm{NC}-\mathrm{OPE} 2-\mathrm{CC}-\mathrm{CC}$ junctions. 2D histograms of $\mathrm{NC}-\mathrm{OPE} 2-\mathrm{CC}-\mathrm{CC}$ with the terminal groups of (a) $-\mathrm{H}$ and (b) $-\mathrm{AuPPh}_{3}$. The relative intensity around $10^{-4} \sim 10^{-5} \mathrm{G}_{0}$ and $0.0 \sim 0.3 \mathrm{~nm}$ suggests that a significant percentage of traces in Panel a exhibited through space tunneling decay and did not form molecular junctions. ${ }^{22}$ Probability of junction formation: a, $40.5 \%$; b, $15.8 \%$. Number of traces: a, 5105 ; b, 5050. Conditions: $V_{\text {bias, }} 50 \mathrm{mV}$; solvent, mesitylene.
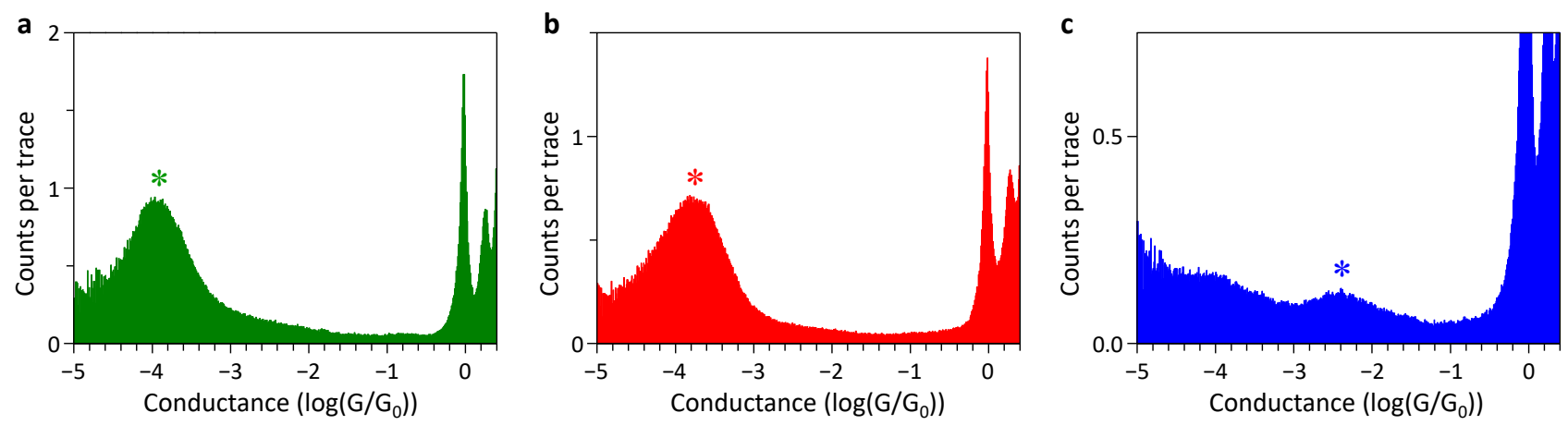

Figure S7. Molecular conductance histograms of (a) NC-OPE2-CC-CC, (b) NC-OPE2-CC, and (c) $\mathrm{NC}-\mathrm{C} 4-\mathrm{CC}$ junctions. All three conductance histograms were constructed from more than 5,000 conductance traces without data selection. The molecular conductance peak is indicated by the asterisk. Conditions: $V_{\text {bias, }} 50 \mathrm{mV}$; solvent, mesitylene. 
To assess the molecular moiety that forms the molecular junctions, we calculated bond dissociation energy for $-\mathrm{CC}-\mathrm{AuPPh}_{3}$. Table $\mathrm{S} 1$ shows that the dissociation energy for $\mathrm{Au}-\mathrm{P}$ is the smallest. The results suggest that, upon the formation of $\mathrm{NC}-\mathrm{OPE} 2-\mathrm{CC}-\mathrm{AuPPh}_{3}$ junctions, the $-\mathrm{PPh}_{3}$ moiety leaves while the $-\mathrm{CC}-\mathrm{Au}$ bond remains intact. This terminal gold becomes an apical gold atom of the gold electrode.

Table S1. Dissociation energy for selected bonds of NC-OPE2-CC-AuPPh.

\begin{tabular}{|c|c|c|c|}
\hline \multirow[b]{2}{*}{ Entry } & \multicolumn{3}{|c|}{ bond dissociation energies $(\mathrm{eV})$} \\
\hline & BLYP & PBW91 & PBE \\
\hline $\mathrm{NC}-\mathrm{OPE} 2-\mathrm{CC} \frac{\hat{s}}{\mathrm{~A}} \mathrm{Au}-\mathrm{PPh}_{3}$ & 7.91366 & 7.57041 & 4.98591 \\
\hline $\mathrm{NC}-\mathrm{OPE} 2-\mathrm{CC}-\mathrm{Au}^{\frac{\hat{S}}{\xi} \mathrm{PPh}_{3}}$ & 3.18107 & 2.90657 & 2.95080 \\
\hline $\mathrm{NC}-\mathrm{OPE} 2-\mathrm{CC}-\mathrm{Au}-\mathrm{P}-\mathrm{Ph}_{2} \frac{\hat{\xi}}{\xi} \mathrm{Ph}$ & 8.23052 & 4.24533 & 4.48566 \\
\hline NC-OPE2-CC-Au-P $\frac{\{}{\xi} 3 \mathrm{Ph}$ & 33.33104 & 33.49777 & 19.25463 \\
\hline
\end{tabular}

${ }^{a}$ Exchange-correlation were three GGA types with BLYP, PBW91 and PBE functional. ${ }^{\mathrm{b}}$ Simulations were performed with the condition of $300 \mathrm{~K}$. ${ }^{\mathrm{c}}$ The symbol $\xi$ represents bond breaking location. 


\section{Peak assignment for transmission spectra}

\section{4-1. Peaks 1-4 in the transmission spectra of NC-OPE2-CC junctions.}

To provide the details for Figure $2 \mathrm{~d}$ of the main text, Figure S8a presents the isosurface plots of the $\mathrm{NC}-\mathrm{OPE} 2-\mathrm{CC}$ junctions to correlate with the gas-phase eigenstates of $\mathrm{NC}-\mathrm{OPE} 2-\mathrm{CCH}$ (Figure S8b). The plots at the upper and lower rows are differed by a 90-degree rotation about the z-axis. The isosurface plots for Peaks 2, 3, and 4 of the transmission spectra (Figure 2a) are similar to the gas-phase HOMO, LUMO, and LUMO+1, respectively. Peak 1 is predominantly contributed by the gold electrodes.

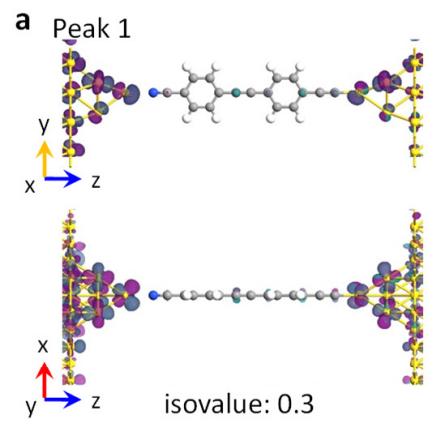

b HOMO-1

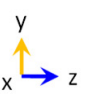

$\stackrel{x}{\mathrm{~A}} \mathrm{z}$

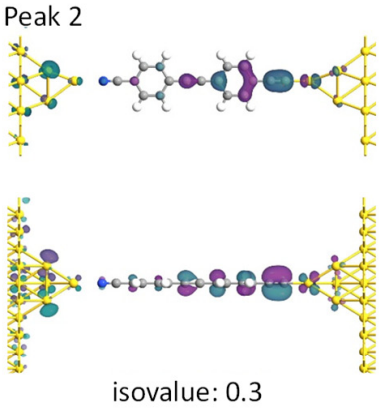

HOMO
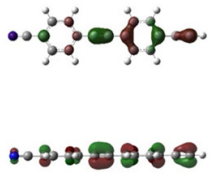

isovalue: 0.05
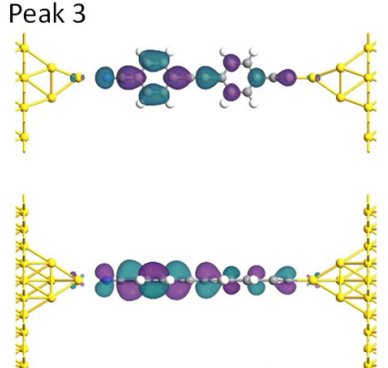

isovalue: 0.7

LUMO

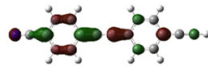

seresest

isovalue: 0.03

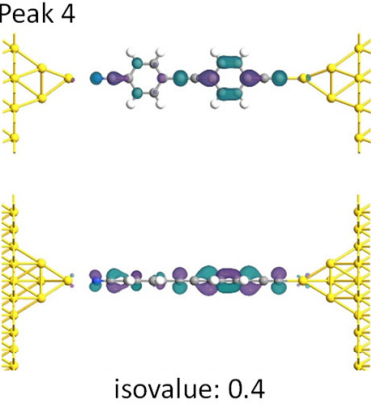

LUMO+1

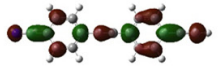

2383808

isovalue: 0.03

Figure S8. Isosurface plots of $\mathrm{NC}-\mathrm{OPE} 2-\mathrm{CC}$ junctions and $\mathrm{NC}-\mathrm{OPE} 2-\mathrm{CCH}$. Isosurface plots for (a) transmission Peaks 1-4 (Figure 2 in the main text) of the NC-OPE2-CC junction with pyramidal electrodes and for (b) the gas-phase eigenstates of $\mathrm{NC}-\mathrm{OPE} 2-\mathrm{CCH}$. Each isosurface are plotted by two viewing angles with a 90 -degree rotation about the $\mathrm{z}$-axis. 


\section{4-2. Orbitals facilitating the electron transport at $-\mathrm{CC}-\mathrm{Au}$ contacts.}

$\boldsymbol{\sigma}$ and $\pi$ characters of the direct $-\mathbf{C C}-\mathbf{A u}$ bonds. The conductance of covalently bonded singlemolecule junctions is found to be superior to their analogues with other contacts. ${ }^{14,22-26}$ The improved conductance is attributed to the carbon-electrode $\sigma$ bond. ${ }^{14,22-27}$ In this present study of ethynyl-based $-\mathrm{CC}-\mathrm{Au}$ interface, there are $\sigma$ and $\pi$ characters. Figure S8a shows the transmission spectra of $\mathrm{NC}-\mathrm{C} 4-\mathrm{CC}$ junctions. The filled and open circles indicate where $-\mathrm{CC}-\mathrm{Au} \sigma$ characters for $\sigma+d_{\mathrm{z}^{2}}$ bonding and $\sigma-d_{\mathrm{z}^{2}}$ antibonding orbitals are located, respectively. The $\pi$ character $\left(\pi_{\mathrm{x}}-d_{\mathrm{xz}}\right)$ at $-1.1 \mathrm{eV}$ is the FMO of the transmission spectra. The results suggest that the $\sigma$ character stabilizes the molecular junction and the $\pi$ character is the dominant transport pathway.

The degenerate $\pi_{\mathbf{x}}$ and $\pi_{\mathbf{y}}$ orbitals of $\mathbf{N C}-\mathbf{C 4}-\mathbf{C C H}$. In the PDOS plots (e.g., Figure $3 \mathrm{~b}$ and S9b) for $\mathrm{NC}-\mathrm{OPE} 2-\mathrm{CC}$ junctions, there are two distinct and close peaks of $\pi_{\mathrm{x}}$ and $\pi_{\mathrm{y}}$ orbitals. The peak for the $\pi_{\mathrm{y}}$ orbital appears insignificant in the transmission spectra. This is attributed to the orthogonality of $-\mathrm{CC}-\mathrm{Au} \pi_{\mathrm{y}}$ orbitals to the aromatic $\pi_{\mathrm{y}}$ backbone. Intriguingly, similar features are observed for butane backbone. Specifically, the FMO of the spectrum (Figure S9a) is contributed by $\pi_{x}$ orbital only. Figure S9c shows that, although the two $\pi$ orbitals are degenerate, $\pi_{x}$ orbital couples with the methylene chain better than $\pi_{\mathrm{y}}$ orbital.

a

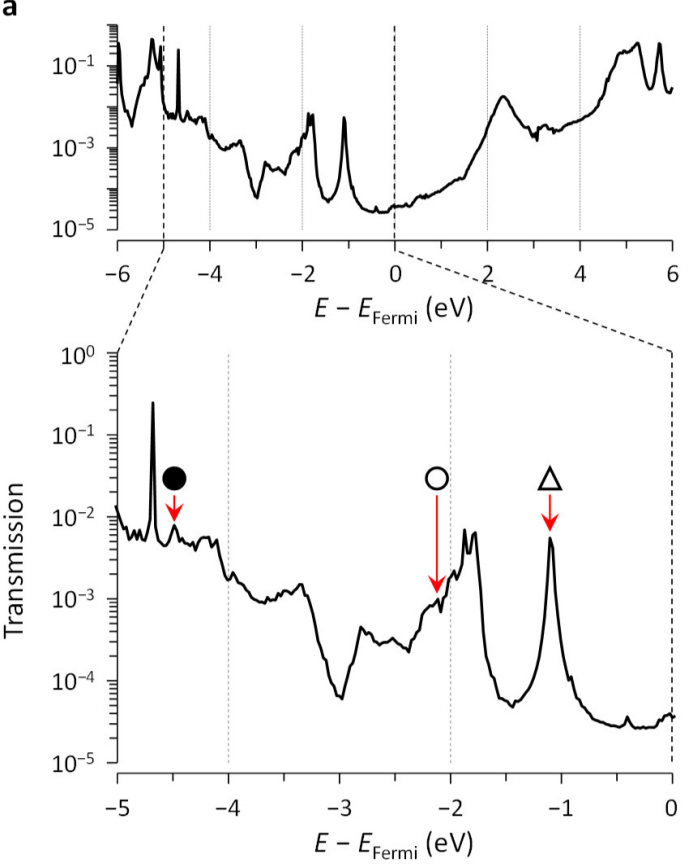

b.

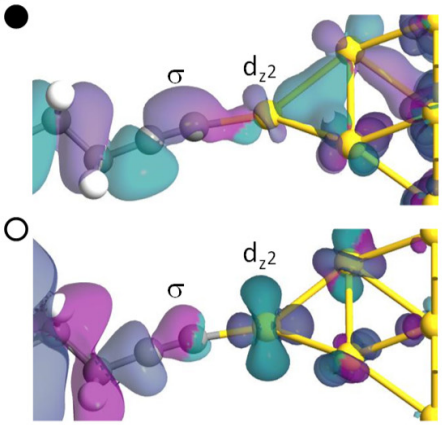

$\triangle$

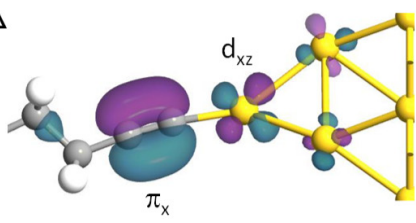

c
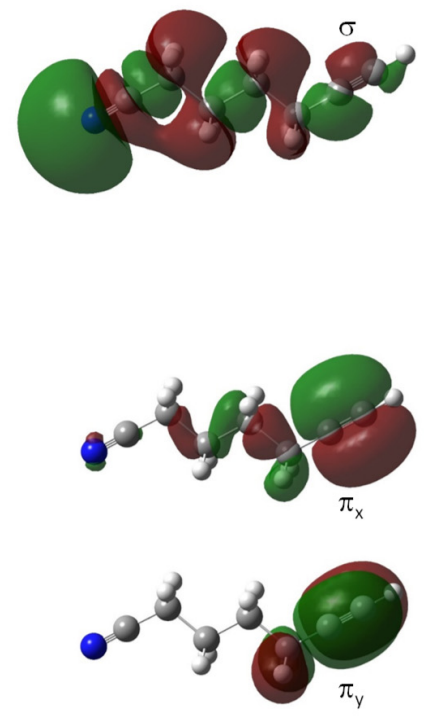

Figure S9. Transmission spectra and isosurface plots of $\mathrm{NC}-\mathrm{C} 4-\mathrm{CC}$. (a) Transmission spectra and (b) portions of the isosurface plots of $\mathrm{NC}-\mathrm{C} 4-\mathrm{CC}$ junctions. (c) Isosurface plots of $\mathrm{NC}-\mathrm{C} 4-\mathrm{CCH}$ in the gas phase. In the free-space calculations, the $\pi_{\mathrm{x}}$ and $\pi_{\mathrm{y}}$ orbitals are degeneracy. However, in the transmission spectrum, the major contribution is through the $\pi_{\mathrm{x}}$ orbital due to the coupling with the methylene backbone. Symbols and energy of mixing orbitals: $\bullet, \sigma+d_{\mathrm{z}^{2}},-4.5 \mathrm{eV} ; \circ, \sigma-d_{\mathrm{z}^{2}},-2.1 \mathrm{eV} ; \triangle, \pi_{\mathrm{x}}-d_{\mathrm{xz}},-1.1 \mathrm{eV}$. 


\section{Simulated junction characteristics on pyramidal, stepped, and planar electrodes.}

The electrode geometries of pyramidal, stepped, and planar $\mathrm{Au}(111)$ are optimized and utilized for the junction structures for the model compounds. Simulated results of this study and literature reports $^{22,28-30}$ show that the junction conductance is dependent on how the contacted gold atom is arranged with the neighboring atoms on the electrode. Due to the space limitation of the main text, only selective data are presented in Figures 2, 3, and 5. This section provides detailed information.

a

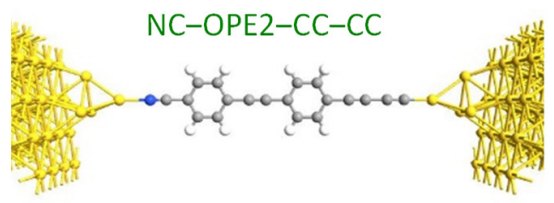

b

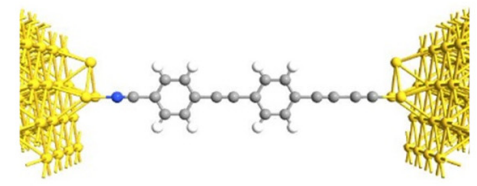

C

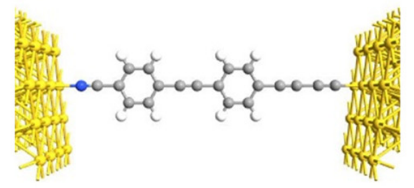

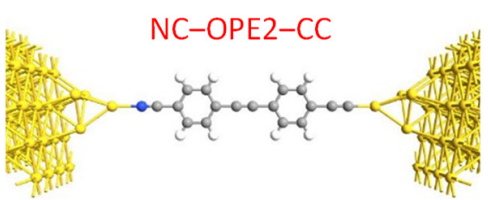
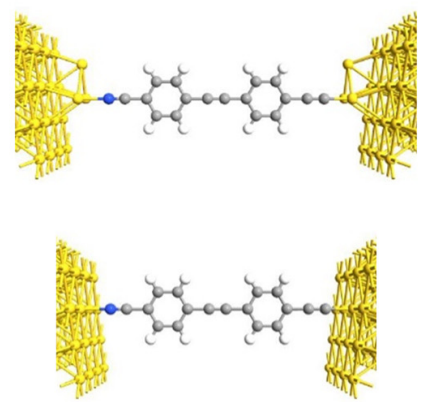
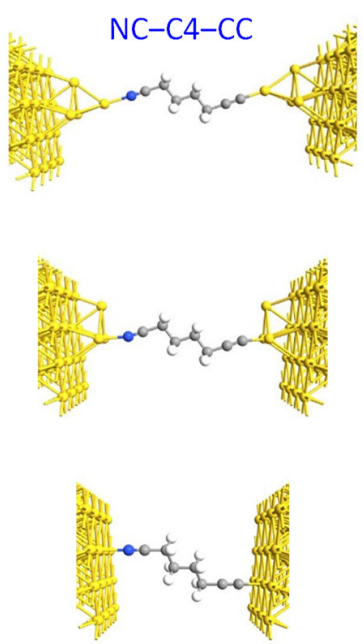

Figure S10. Three electrode geometries for the simulations of junction characteristics. Initial structures for the optimization of ethynyl-based model compounds bridged between a pair of (a) pyramidal, (b) stepped, and (c) planar $\mathrm{Au}(111)$ electrodes.
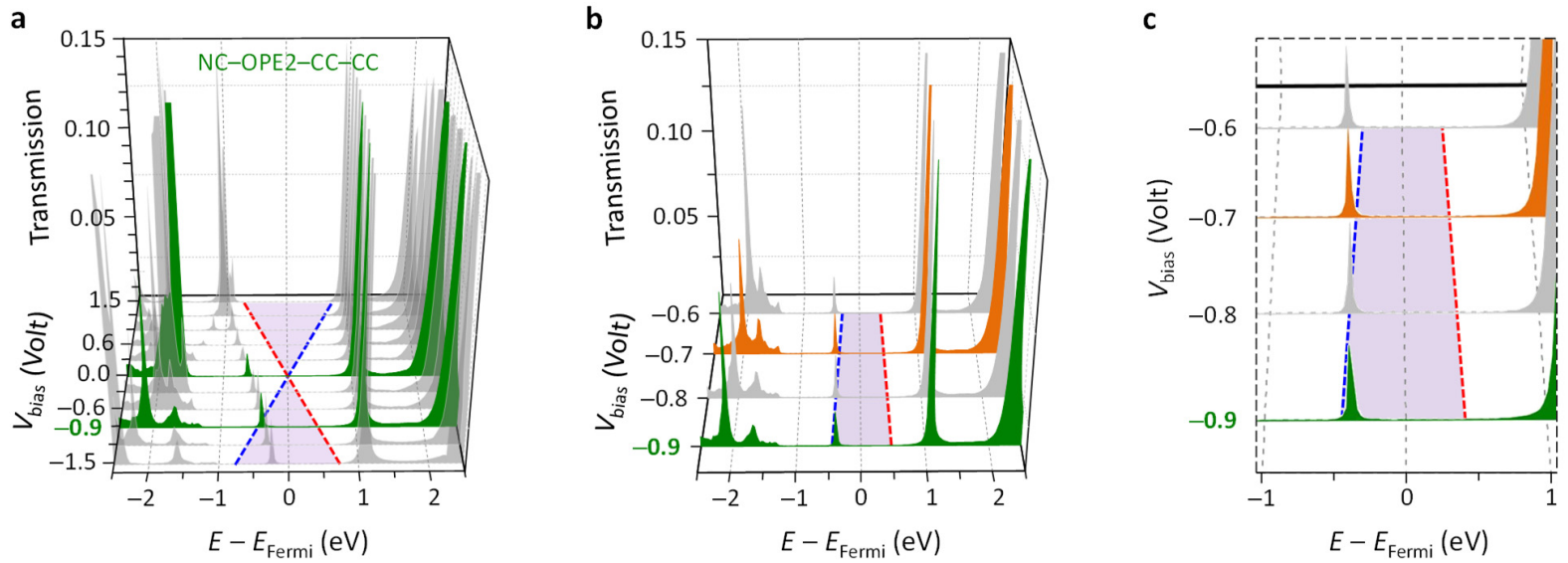

Figure S11. Zoomed transmission spectra at $V_{\text {bias }}=-0.7 \mathrm{~V}$ for $\mathrm{NC}-\mathrm{OPE} 2-\mathrm{CC}-\mathrm{CC}$ junctions $\left(V_{\text {bias }}=\right.$

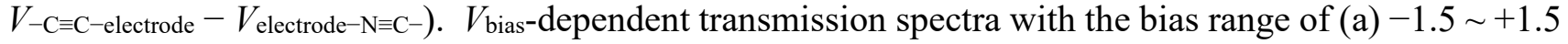
$\mathrm{V}$ at $0.3 \mathrm{~V}$ intervals and $(\mathrm{b}, \mathrm{c})-0.6 \sim-0.9 \mathrm{~V}$ at $0.1 \mathrm{~V}$ intervals to manifest that Peak 2 of Figure $2 \mathrm{~b}$ is involved in the potential window at $V_{\text {bias }}$ around $-0.7 \mathrm{~V}$. The red and blue dash lines are the voltage applied to the $-\mathrm{CC}$-electrode and $-\mathrm{CN}$-electrode terminals, respectively. The intersect begins at $-0.7 \mathrm{~V}$ (orange color) and Peak 2 moves inside the bias window at $-0.9 \mathrm{~V}$ (green color). Hence, the onset potential for this junction is smaller than the other model compounds. Pyramidal electrodes were used for the simulations. 

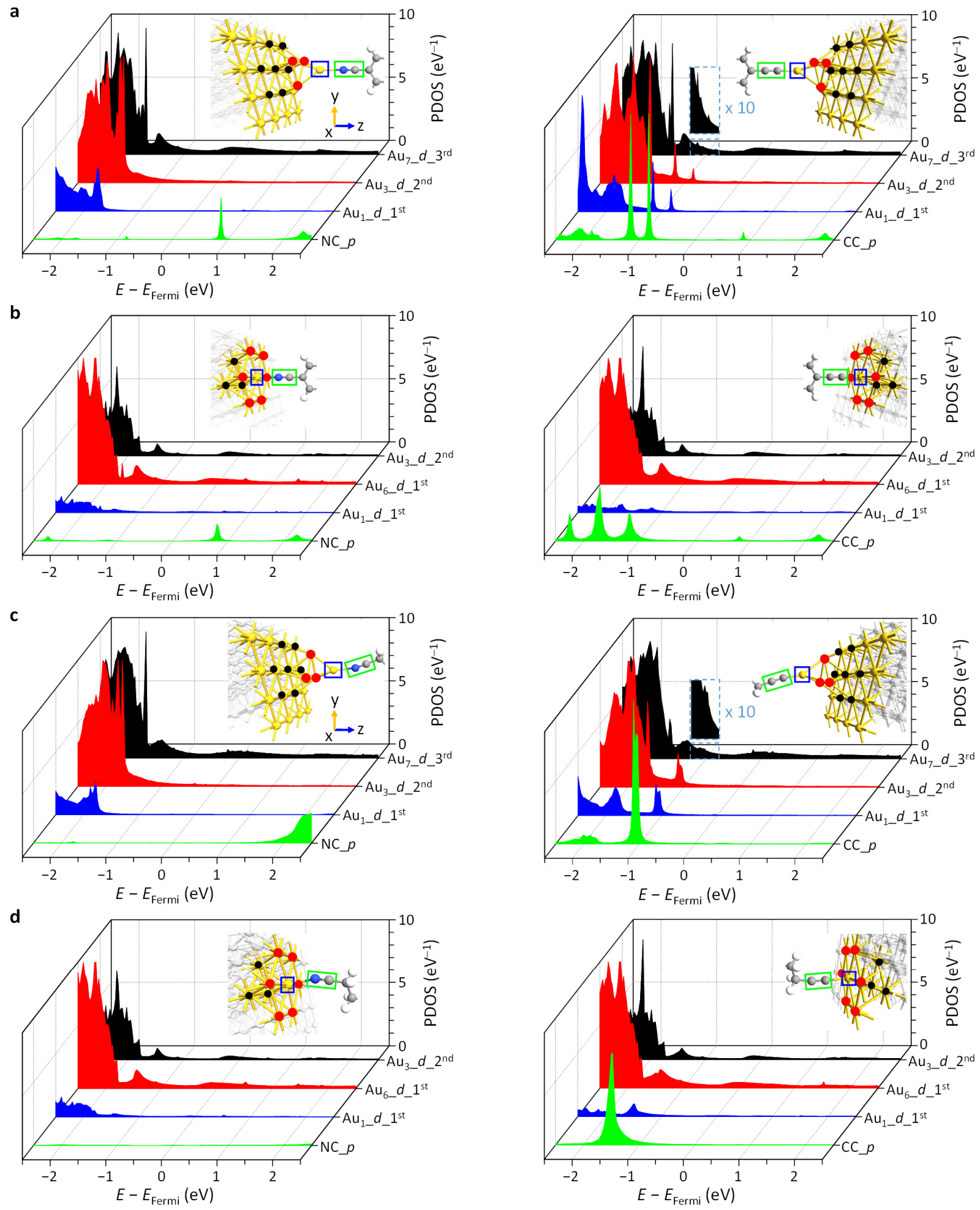

Figure S12. PDOS plots for NC-OPE2-CC and NC-C4-CC junctions. (a,b) NC-OPE2-CC and (c,d) $\mathrm{NC}-\mathrm{C} 4-\mathrm{CC}$ junctions are simulated with pyramidal and planar $\mathrm{Au}(111)$ electrodes. Left and right columns illustrate $\mathrm{Au}-\mathrm{NC}-$ and $-\mathrm{CC}-\mathrm{Au}$ contacts, respectively. For pyramidal electrodes, the orbital mixing of $-\mathrm{CC}-\mathrm{Au}$ contacts involves gold atoms at the second and third layers. For planar electrodes, the $-\mathrm{CC}-\mathrm{Au}$ ligand-metal orbital mixing is contributed only from the contacted $\mathrm{Au}$ atom. Peak positions: $\left(\pi_{\mathrm{y}}-d, \pi_{\mathrm{x}}-d\right)$ of $-\mathrm{CC}-\mathrm{Au}: \mathrm{a},-1.15 \mathrm{eV},-0.83 \mathrm{eV} ; \mathrm{b},-1.70 \mathrm{eV},-1.18 \mathrm{eV} ; p$ orbital of NC: a, $0.88 \mathrm{eV} ; \mathrm{b}$, $0.80 \mathrm{eV} ; \mathrm{c}, 2.48 \mathrm{eV} ; \mathrm{d},>2.5 \mathrm{eV}$. The color codes and notation are the same as Figure 3. 
a
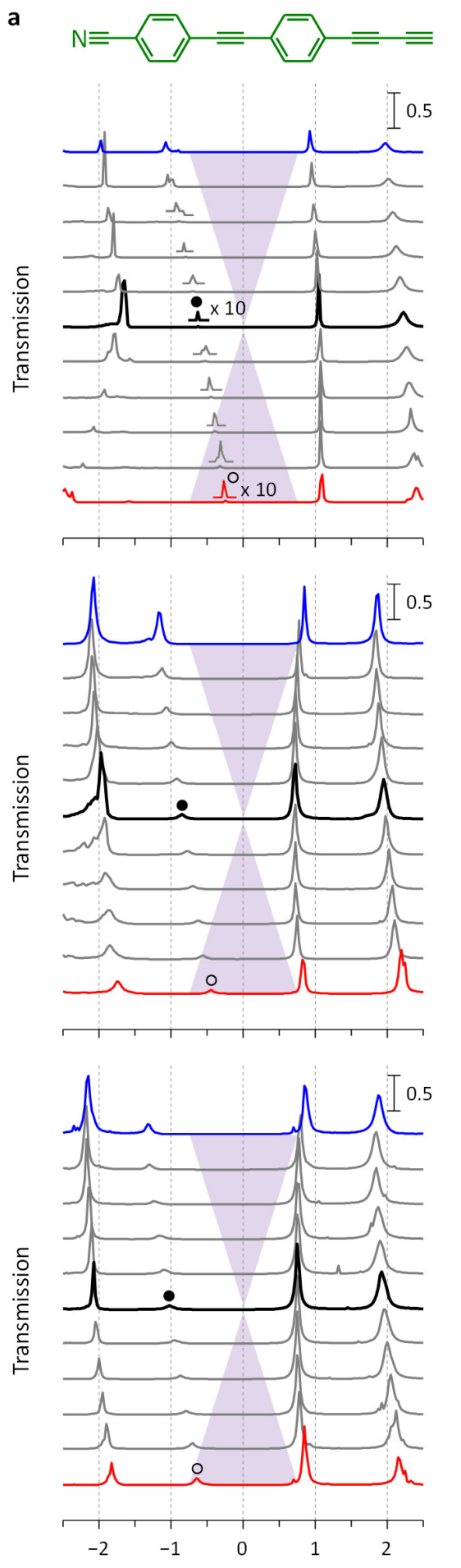

b
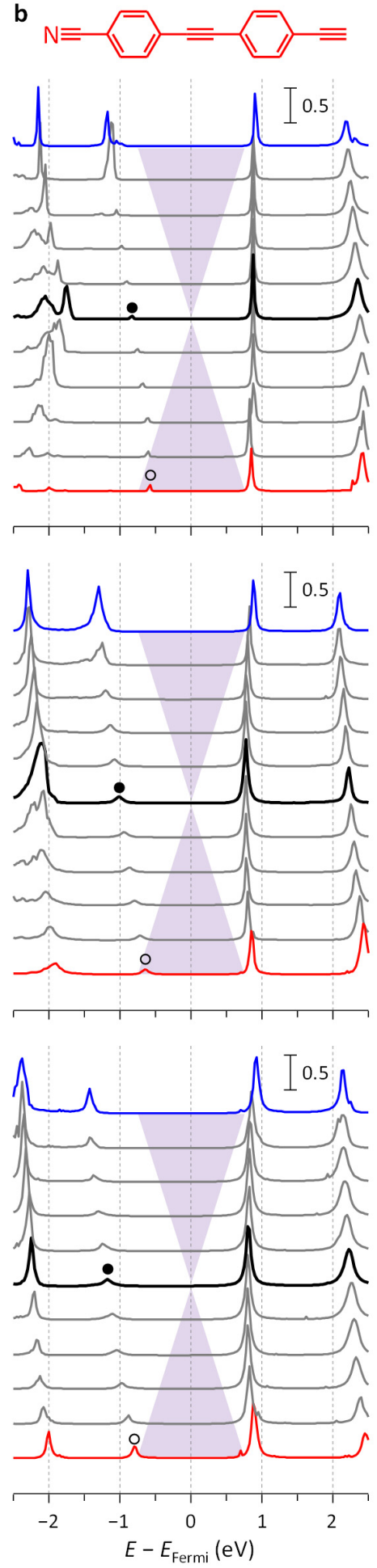

C $\mathrm{N} \equiv \mathrm{C} \sim \sim \mathrm{C} \equiv \mathrm{C}$
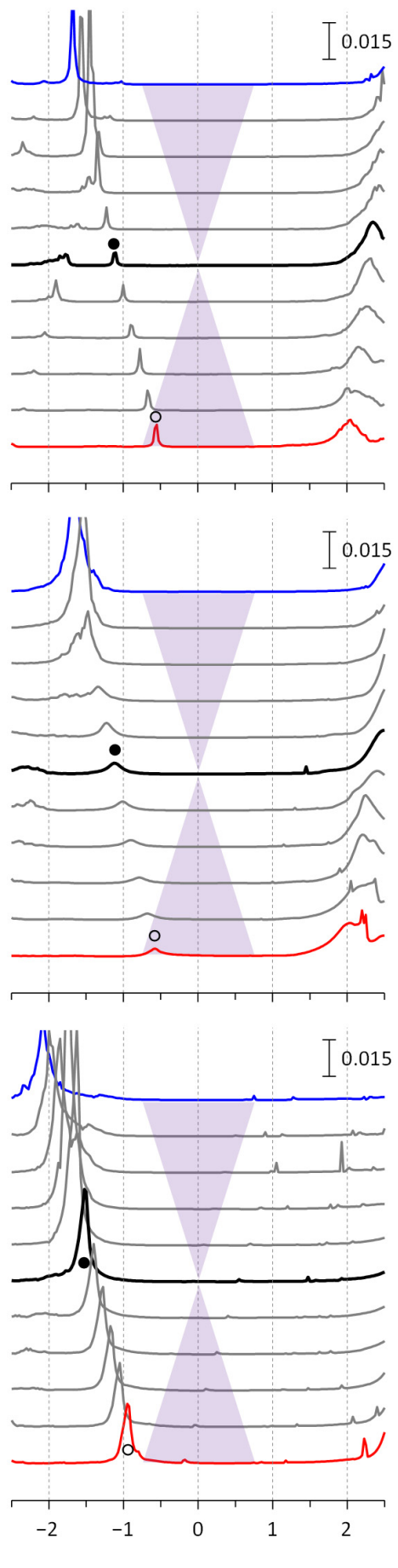

Figure S13. Effect of electrode geometry and $V_{\text {bias }}$ on the shift of $-\mathrm{CC}-\mathrm{Au} \pi_{\mathrm{x}}-d$ orbital. $V_{\text {bias }}-\mathrm{dependent}$ transmission spectra for (a) NC-OPE2-CC-CC, (b) NC-OPE2-CC, and (c) NC-C4-CC bridged between a pair of (upper) pyramidal, (middle) stepped, and (bottom) planar $\mathrm{Au}(111)$ electrodes. The spectra are calculated with the bias applied in the range of $-1.5 \mathrm{~V}$ (red trace) $\leq V_{\text {bias }} \leq+1.5 \mathrm{~V}$ (blue trace) at $0.3 \mathrm{~V}$ intervals. The shaded areas are the bias window. The filled $(\bullet)$ and open $(\circ)$ circles indicate the positions of Peak 2 at zero bias and $-1.5 \mathrm{~V}$, respectively. Pyramidal electrodes make Peak 2 moving inside the potential window at the smallest $V_{\text {bias, }}$ suggesting the strongest pinning effect and the smallest onset potential for rectification (Figure S14). 
a
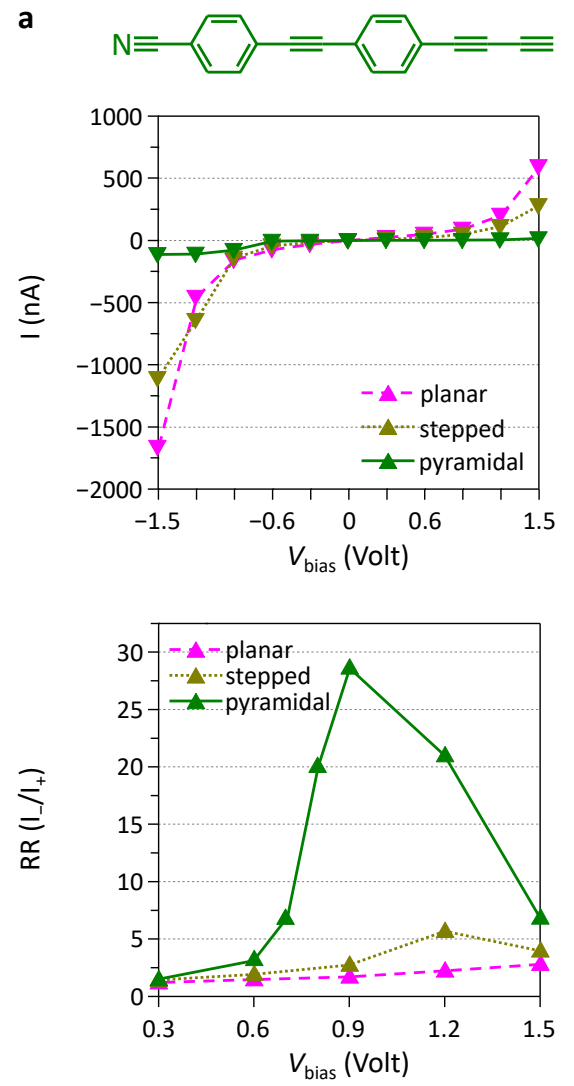

b
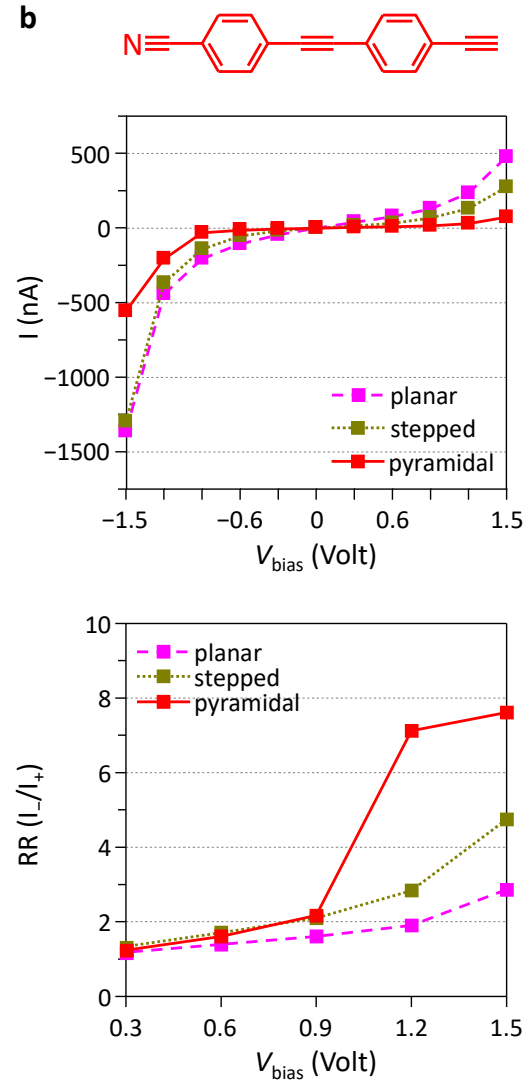

c
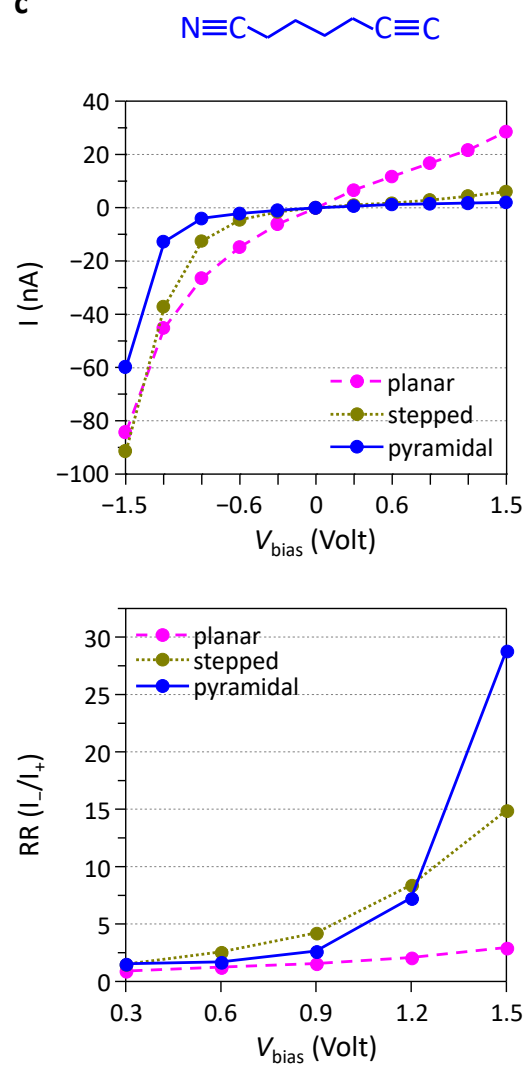

Figure S14. Effect of electrode geometry on simulated $i-V_{\text {bias }}$ curves and rectification ratios. (Upper) simulated $i-V_{\text {bias }}$ curves and (lower) rectification ratios of (a) $\mathrm{NC}-\mathrm{OPE} 2-\mathrm{CC}-\mathrm{CC}$, (b) NC-OPE2-CC, and (c) NC-C4-CC bridged in the junctions of pyramidal, stepped, and planar electrodes. The currents are obtained by the integration of the transmission spectra within the bias window. The currents obtained from pyramidal junctions are smaller than those from the other two electrode geometries $\left(i_{\text {pyramidal }}<i_{\text {stepped }}\right.$ $\leq i_{\text {planar). }}$. Pyramidal electrodes also result in the smallest onset potentials for the rectification. For compounds with OPE2-moieties, the tail of the LUMO peak also moves into the potential window at a both the more positive and more negative $V_{\text {bias }}$ (see Figure S13a and 13b). The increase in currents at both positive and negative bias limits the RR values. The RR curves at pyramidal junctions (i.e., the green, red, and blue curves of the lower panels) for the model compounds are identical to those of Figure $2 \mathrm{f}$ in the main text. 


\section{Data processing for the experimental $i-V_{\text {bias }}$ curves.}

Removal of $\boldsymbol{i}-\boldsymbol{V}_{\text {bias }}$ curves from broken junctions. Figure 4 in the main text presents $i-V_{\text {bias }}$ curves pooled after the removal of those with broken junctions. For comparison, Figure S15a shows the raw $i-V_{\text {bias }}$ curves of $\mathrm{NC}-\mathrm{OPE} 2-\mathrm{CC}-\mathrm{CC}$ junctions and is employed to describe the data selection.

The junction current presumably increases with the applied bias. For junctions broken upon the $V_{\text {bias }}$ sweeping, the current at a larger bias is significantly smaller. Accordingly, to find curves with broken junctions, the integrated $i-V_{\text {bias }}$ areas are compared between neighboring bias segments. The dashed lines in Figure S14a illustrate 4 equally spaced bias intervals from $0 \mathrm{~V}$ to $+1.5 \mathrm{~V}$ (and also 4 quarters for the negative bias of $0 \sim-1.5 \mathrm{~V}$ ). The removal of traces was carried out by an algorithm. The resulted $i-V_{\text {bias }}$ curves were plotted to give Figure S15b in which the contribution of low conductance traces at $\left|V_{\text {bias }}\right|>$ $0.3 \mathrm{~V}$ and $\mathrm{I}<10^{-9}$ ampere (viz., near the background level) becomes less significant than that in Figure S14a. For each compound, about $20 \%$ of the $i-V_{\text {bias }}$ curves did not pass the selection criteria.

Electrode geometry, current levels, and rectification ratios. The upper panels of Figure $5 \mathrm{a}$ in the main text show the $i-V_{\text {bias }}$ curves of $\mathrm{NC}-\mathrm{OPE} 2-\mathrm{CC}-\mathrm{CC}$ junctions with relatively large (left panel) and small currents (right panel) based on the simulation results (Figure S14) that planar electrodes yield the largest current and pyramidal ones the smallest. Hence, curves with larger currents are more similar to those of planar electrodes than those of pyramidal electrodes; curves with smaller currents show more characteristics of pyramidal electrodes than those of planar ones.

Figure S14b denotes I, II and III, IV along the y-axes to indicate regimes of relatively large and small currents, respectively. The abovementioned large and small currents were arbitrarily set as the top and bottom $12 \%$ of the curves. Figure S15c displays three $i-V_{\text {bias }}$ curves from Regime IV and their rectified features are consistent with the assumption that curves with smaller currents more likely exhibit features of pyramidal electrodes. Figure S15d shows those obtained from the four regimes. The upper panels of Figure 5a was plotted by reversing the bias for curves in Regimes II and III which were then pooled together with those of I and IV, respectively. 

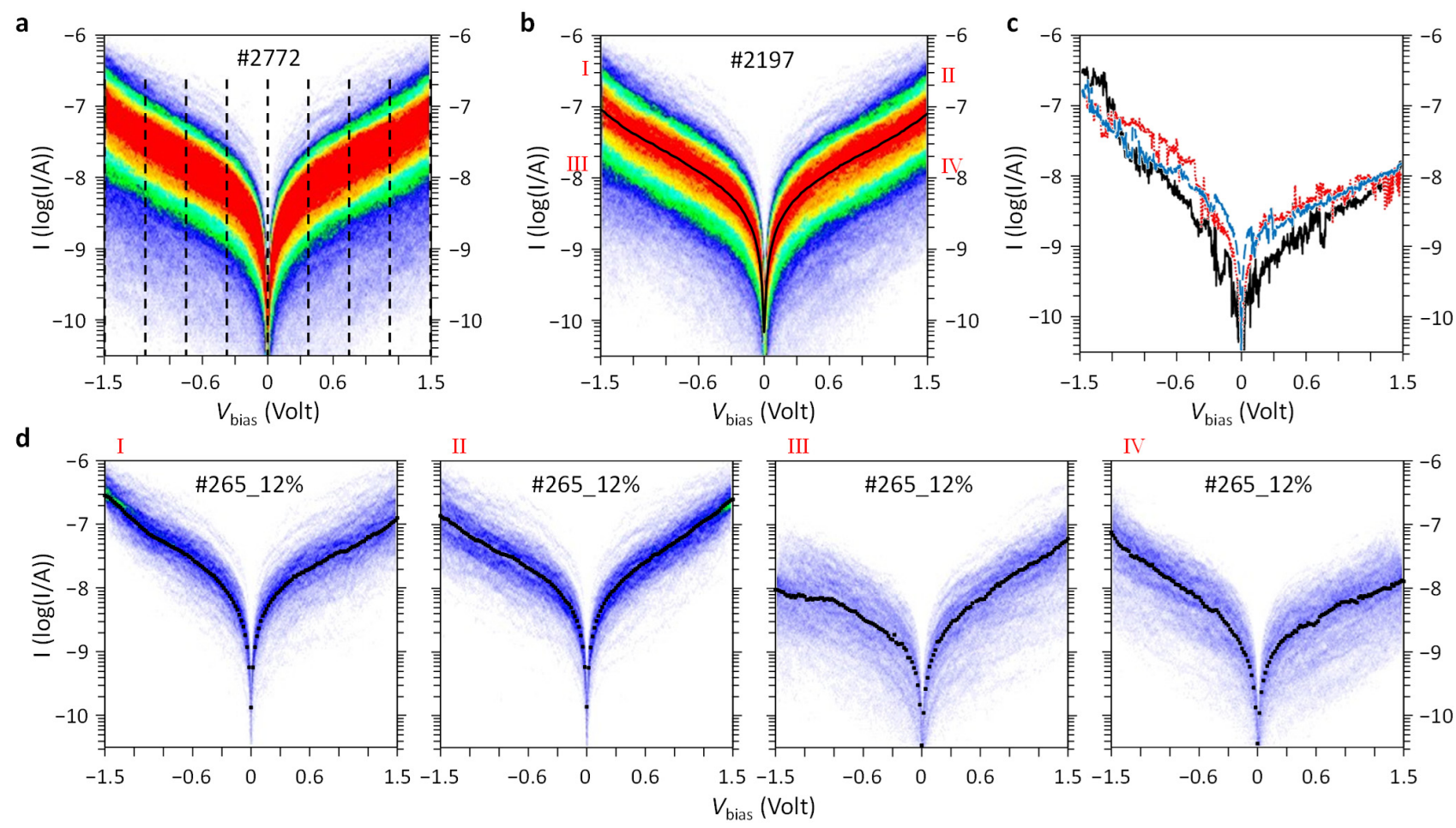

Figure S15. Treatment of the experimental $i-V_{\text {bias }}$ curves of NC-OPE2-CC-CC. (a) Overlaid raw $i-V_{\text {bias }}$ curves. The dashed lines divide the bias range into 8 segments at $0.375 \mathrm{~V}$ intervals. (b) Overlaid $i-V_{\text {bias }}$ curves after the removal of curves with broken junctions. The black trace shows the trajectory of the most probable curve obtained by Gaussian fitting at $20 \mathrm{mV}$ intervals. Regimes I, II and III, IV represent the largest and smallest $12 \%$ of current at $\pm 1.5 \mathrm{~V}$. (c) Typical $i-V_{\text {bias }}$ curves from Regime IV. (d) $i-V_{\text {bias }}$ curves from the four regimes. 


\section{References:}

(1) Koga, J.; Tsuji, Y.; Yoshizawa, K. Orbital Control of Single-Molecule Conductance Perturbed by $\Pi$-Accepting Anchor Groups: Cyanide and Isocyanide. J. Phys. Chem. C 2012, 116, 20607-20616.

(2) Kinoshita, K.; Kawakami, T.; Morita, Y.; Saito, T.; Yamanaka, S.; Okumura, M.; Yamaguchi, K. Theoretical Studies on the Magnetic and Conductive Properties of Crystals Containing Open-Shell Trioxotriangulene Radicals. Bull. Chem. Soc. Jpn. 2015, 89, 315-333.

(3) Nozaki, D.; Toher, C. Is the Antiresonance in Meta-Contacted Benzene Due to the Destructive Superposition of Waves Traveling Two Different Routes around the Benzene Ring? J. Phys. Chem. C 2017, 121, 11739-11746.

(4) Khairul, W. M.; Albesa-Jové, D.; Yufit, D. S.; Al-Haddad, M. R.; Collings, J. C.; Hartl, F.; Howard, J. A.; Marder, T. B.; Low, P. J. The Syntheses, Structures and Redox Properties of Phosphine-Gold(I) and Triruthenium-Carbonyl Cluster Derivatives of Tolans. Inorg. Chim. Acta 2008, 361, 1646-1658.

(5) Frisch, M. J.; Trucks, G. W.; Schlegel, H. B.; Scuseria, G. E.; Robb, M. A.; Cheeseman, J. R., et al. Gaussian 09 Rev. A.01, Wallingford, CT, 2016.

(6) Smidstrup, S.; Stradi, D.; Wellendorff, J.; Khomyakov, P. A.; Vej-Hansen, U. G.; Lee, M.-E.; Ghosh, T.; Jónsson, E.; Jónsson, H.; Stokbro, K. First-Principles Green's-Function Method for Surface Calculations: A Pseudopotential Localized Basis Set Approach. Phys. Rev. B 2017, 96, 195309.

(7) Brandbyge, M.; Mozos, J.-L.; Ordejón, P.; Taylor, J.; Stokbro, K. Density-Functional Method for Nonequilibrium Electron Transport. Phys. Rev. B 2002, 65, 165401.

(8) Perdew, J. P.; Burke, K.; Ernzerhof, M. Generalized Gradient Approximation Made Simple. Phys. Rev. Lett. 1996, 77, 3865.

(9) Van Dyck, C.; Ratner, M. A. Molecular Rectifiers: A New Design Based on Asymmetric Anchoring Moieties. Nano Lett. 2015, 15, 1577-1584.

(10) Liu, H.-T.; Xiong, X.-G.; Dau, P. D.; Wang, Y.-L.; Huang, D.-L.; Li, J.; Wang, L.-S. Probing the Nature of Gold-Carbon Bonding in Gold-Alkynyl Complexes. Nat. Commun. 2013, 4, 2223.

(11) Lin, G.-M.; Lin, C.-H.; Peng, H. H.; Hsiao, H.; Wang, T.-H.; Ho, C.-H.; Hsu, H.-F.; Chen, C.-h. Effect of the Chemical Potentials of Electrodes on Charge Transport across Molecular Junctions. J. Phys. Chem. C 2019, 123, 22009-22017.

(12) Ting, T.-C.; Hsu, L.-Y.; Huang, M.-J.; Horng, E.-C.; Lu, H.-C.; Hsu, C.-H.; Jiang, C.-H.; Jin, B.Y.; Peng, S.-M.; Chen, C.-h. Energy-Level Alignment for Single-Molecule Conductance of Extended Metal-Atom Chains. Angew. Chem. Int. Ed. 2015, 54, 15734-15738.

(13) Zang, Y.; Pinkard, A.; Liu, Z.-F.; Neaton, J. B.; Steigerwald, M. L.; Roy, X.; Venkataraman, L. Electronically Transparent Au-N Bonds for Molecular Junctions. J. Am. Chem. Soc. 2017, 139, $14845-14848$.

(14) Hong, W.; Li, H.; Liu, S.-X.; Fu, Y.; Li, J.; Kaliginedi, V.; Decurtins, S.; Wandlowski, T. Trimethylsilyl-Terminated Oligo(phenyleneethynylene)s: An Approach to Single-Molecule Junctions with Covalent Au-C Sigma-Bonds. J. Am. Chem. Soc. 2012, 134, 19425-19431.

(15) Hong, W.; Manrique, D. Z.; Moreno-Garcia, P.; Gulcur, M.; Mishchenko, A.; Lambert, C. J.; Bryce, M. R.; Wandlowski, T. Single Molecular Conductance of Tolanes: Experimental and Theoretical Study on the Junction Evolution Dependent on the Anchoring Group. J. Am. Chem. Soc. 2012, 134, 2292-2304.

(16) Miguel, D.; de Cienfuegos, L. A.; Martín-Lasanta, A.; Morcillo, S. P.; Zotti, L. A.; Leary, E.; Bürkle, M.; Asai, Y.; Jurado, R.; Cárdenas, D. J.; Rubio-Bollinger, G.; Agraï, N.; Cuerva, J. M.; González, M. T. Toward Multiple Conductance Pathways with Heterocycle-Based Oligo(phenyleneethynylene) Derivatives. J. Am. Chem. Soc. 2015, 137, 13818-13826.

(17) Batra, A.; Meisner, J. S.; Darancet, P.; Chen, Q.; Steigerwald, M. L.; Nuckolls, C.; Venkataraman, L. Molecular Diodes Enabled by Quantum Interference. Faraday Discuss. 2014, 174, 79-89. 
(18) Koepf, M.; Koenigsmann, C.; Ding, W.; Batra, A.; Negre, C. F.; Venkataraman, L.; Brudvig, G. W.; Batista, V. S.; Schmuttenmaer, C. A.; Crabtree, R. H. Controlling the Rectification Properties of Molecular Junctions through Molecule-Electrode Coupling. Nanoscale 2016, 8, 16357-16362.

(19) Batra, A.; Darancet, P.; Chen, Q.; Meisner, J. S.; Widawsky, J. R.; Neaton, J. B.; Nuckolls, C.; Venkataraman, L. Tuning Rectification in Single-Molecular Diodes. Nano Lett. 2013, 13, 6233-6237.

(20) Li, Y.; Buerkle, M.; Li, G.; Rostamian, A.; Wang, H.; Wang, Z.; Bowler, D. R.; Miyazaki, T.; Xiang, L.; Asai, Y. Gate Controlling of Quantum Interference and Direct Observation of AntiResonances in Single Molecule Charge Transport. Nat. Mater. 2019, 18, 357-363.

(21) Bai, J.; Daaoub, A.; Sangtarash, S.; Li, X.; Tang, Y.; Zou, Q.; Sadeghi, H.; Liu, S.; Huang, X.; Tan, Z. Anti-Resonance Features of Destructive Quantum Interference in Single-Molecule Thiophene Junctions Achieved by Electrochemical Gating. Nat. Mater. 2019, 18, 364-369.

(22) Bejarano, F.; Olavarria-Contreras, I. J.; Droghetti, A.; Rungger, I.; Rudnev, A.; Gutiérrez, D.; Mas-Torrent, M.; Veciana, J.; van der Zant, H. S.; Rovira, C. Robust Organic Radical Molecular Junctions Using Acetylene Terminated Groups for C-Au Bond Formation. J. Am. Chem. Soc. 2018, 140, 1691-1696.

(23) Chen, W. B.; Widawsky, J. R.; Vazquez, H.; Schneebeli, S. T.; Hybertsen, M. S.; Breslow, R.; Venkataraman, L. Highly Conducting П-Conjugated Molecular Junctions Covalently Bonded to Gold Electrodes. J. Am. Chem. Soc. 2011, 133, 17160-17163.

(24) Cheng, Z. L.; Skouta, R.; Vazquez, H.; Widawsky, J. R.; Schneebeli, S.; Chen, W.; Hybertsen, M. S.; Breslow, R.; Venkataraman, L. In Situ Formation of Highly Conducting Covalent $\mathrm{Au}-\mathrm{C}$ Contacts for Single-Molecule Junctions. Nat. Nanotechnol. 2011, 6, 353-357.

(25) Fu, Y.; Chen, S.; Kuzume, A.; Rudnev, A.; Huang, C.; Kaliginedi, V.; Baghernejad, M.; Hong, W.; Wandlowski, T.; Decurtins, S. Exploitation of Desilylation Chemistry in Tailor-Made Functionalization on Diverse Surfaces. Nat. Commun. 2015, 6, 6403.

(26) Huang, C.; Chen, S.; Ørnsø, K. B.; Reber, D.; Baghernejad, M.; Fu, Y.; Wandlowski, T.; Decurtins, S.; Hong, W.; Thygesen, K. S. Controlling Electrical Conductance through a ПConjugated Cruciform Molecule by Selective Anchoring to Gold Electrodes. Angew. Chem. Int. Ed. 2015, 54, 14304-14307.

(27) Ma, G. H.; Shen, X.; Sun, L. L.; Zhang, R. X.; Wei, P.; Sanvito, S.; Hou, S. M. Low-Bias Conductance of Single Benzene Molecules Contacted by Direct $\mathrm{Au}-\mathrm{C}$ and $\mathrm{Pt}-\mathrm{C}$ Bonds. Nanotechnology 2010, 21, 495202.

(28) Olavarria-Contreras, I. J.; Perrin, M. L.; Chen, Z.; Klyatskaya, S.; Ruben, M.; van der Zant, H. S. $\mathrm{C}-\mathrm{Au}$ Covalently Bonded Molecular Junctions Using Nonprotected Alkynyl Anchoring Groups. J. Am. Chem. Soc. 2016, 138, 8465-8469.

(29) Sugita, Y.; Taninaka, A.; Yoshida, S.; Takeuchi, O.; Shigekawa, H. The Effect of Nitrogen LonePair Interaction on the Conduction in a Single-Molecule Junction with Amine-Au Bonding. Sci. Rep. 2018, 8, 5222.

(30) Zhao, Z.; Liu, R.; Mayer, D.; Coppola, M.; Sun, L.; Kim, Y.; Wang, C.; Ni, L.; Chen, X.; Wang, M. Shaping the Atomic-Scale Geometries of Electrodes to Control Optical and Electrical Performance of Molecular Devices. Small 2018, 14, 1703815. 